\title{
GLOBAL EXISTENCE AND ASYMPTOTIC BEHAVIOUR OF THE SOLUTION TO THE SYSTEM IN ONE-DIMENSIONAL NONLINEAR THERMOVISCOELASTICITY
}

\author{
BY \\ YUMING QIN \\ Department of Mathematics, Henan University, Kaifeng 475001, P.R. China
}

\begin{abstract}
This paper is concerned with the global existence, uniqueness, and asymptotic behavior, as time tends to infinity, of the solution to the system in nonlinear one-dimensional thermoviscoelasticity. Our results show that the global solution approaches to the solution in the $H^{1}$ norm to the corresponding stationary problem, as time tends to infinity.
\end{abstract}

1. Introduction. This paper is concerned with the global existence, uniqueness, and asymptotic behavior, as time tends to infinity, of the solution to the system in nonlinear one-dimensional thermoviscoelasticity. The referential (Lagrangian) form of the conservation laws of mass, momentum, and energy for a one-dimensional material with the reference density $\rho_{0}=1$ is

$$
\begin{aligned}
u_{t}-v_{x} & =0 \\
v_{t}-\sigma_{x} & =0 \\
\left(e+\frac{v^{2}}{2}\right)_{t}-(\sigma v)_{x}+\tilde{q}_{x} & =0
\end{aligned}
$$

and the second law of thermodynamics is expressed by the Clausius-Duhem inequality

$$
\eta_{t}+\left(\frac{\tilde{q}}{\theta}\right)_{x} \geq 0
$$

\footnotetext{
Received January 28, 1999.

2000 Mathematics Subject Classification. Primary 74H40, 35B40, 35Q72, 74D10, 74F05.

The work was supported in part by the grant from Henan Science and Technology Commission and Henan Education Commission.

The work was finished when the author was a Ph.D. candidate in Institute of Mathematics, Fudan University.

E-mail address: qinyuming@mail.henu.edu.cn

Current address: National Laboratory for Scientific Computation, Rua Getulio Vargas 333, Quitandinha 25651-070, Petropolis-RJ, Brazil

Current E-mail address: yuming@lncc.br.
} 
Here subscripts indicate partial differentiations, $u, v, \sigma, e, \tilde{q}, \eta$, and $\theta$ denote the deformation gradient, velocity, stress, internal energy, heat flux, specific entropy, and temperature, respectively. We consider the problem (1.1)-(1.3) in the region $\{0 \leq x \leq 1, t \geq 0\}$ under the initial conditions

$$
u(x, 0)=u_{0}(x), \quad v(x, 0)=v_{0}(x), \quad \theta(x, 0)=\theta_{0}(x) \quad \text { on }[0,1]
$$

and the boundary conditions of the form

$$
\sigma(0, t)=\gamma v(0, t), \quad \sigma(1, t)=-\gamma v(1, t), \quad \theta(0, t)=\theta(1, t)=T_{0},
$$

where $\gamma=0$ or $\gamma=1$, and $T_{0}>0$ is the reference temperature. The boundary condition (1.6) with $\gamma=1$, boundary damping, represents that the endpoints of the interval $[0,1]$ are connected to some sort of dash pot.

For one-dimensional homogeneous, thermoviscoelastic materials, $e, \sigma, \eta$, and $\tilde{q}$ are given by the constitutive relations (see [1])

$$
e=e(u, \theta), \quad \sigma=\sigma\left(u, \theta, v_{x}\right), \quad \eta=\eta(u, \theta), \quad \tilde{q}=\tilde{q}\left(u, \theta, \theta_{x}\right)
$$

which, in order to be consistent with (1.4), must satisfy

$$
\begin{gathered}
\sigma(u, \theta, 0)=\Psi_{u}(u, \theta), \quad \eta(u, \theta)=-\Psi_{\theta}(u, \theta), \\
(\sigma(u, \theta, w)-\sigma(u, \theta, 0)) w \geq 0, \quad \tilde{q}(u, \theta, g) g \leq 0
\end{gathered}
$$

where $\Psi=e-\theta \eta$ is the Helmholtz free energy function.

In this paper we assume that $e(u, \theta), p(u, \theta), \mu(u), \sigma\left(u, \theta, v_{x}\right)$, and $k(u, \theta)$ are twice continuously differentiable on $0<u<+\infty$ and $0 \leq \theta<+\infty$ with the constitutive relations

$$
e=e(u, \theta), \quad \sigma=-p(u, \theta)+\mu(u) v_{x}, \quad \tilde{q}=-k(u, \theta) \theta_{x}
$$

where the viscosity $\mu(u)$ satisfies

$$
\mu(u) u \geq \mu_{0}>0, \quad 0<u<\infty,
$$

for some constant $\mu_{0}$. In order to be consistent with (1.4) or (1.8)-(1.9), e must satisfy

$$
e_{u}(u, \theta)=-p(u, \theta)+\theta p_{\theta}(u, \theta) .
$$

Furthermore, we will be concerned with solid-like materials; so we require that $p(u, \theta)$ be compressive for small $u$ and tensile for large $u$, at any temperature, i.e., there are $0<\tilde{u} \leq \tilde{U}<\infty$ such that

$$
\begin{gathered}
p(u, \theta) \geq 0, \quad 0<u<\tilde{u}, \quad 0 \leq \theta<\infty, \\
p(u, \theta) \leq 0, \quad \widetilde{U}<u<\infty, \quad 0 \leq \theta<\infty .
\end{gathered}
$$

Therefore, the assumptions (1.13)-(1.14) imply that there exists a constant $\eta_{0}$ with $\tilde{u} \leq \eta_{0} \leq \widetilde{U}$ such that

$$
p\left(\eta_{0}, T_{0}\right)=0 .
$$

We also require that $p(u, \theta)$ possess the following monotone condition (see [3]):

$$
\begin{aligned}
& -p_{u}\left(u, T_{0}\right)>0, \quad \text { for any } \underline{u} \leq u \leq \bar{U} \quad \text { if } \gamma=0 \text { in (1.6), } \\
& -p_{u}\left(u, T_{0}\right) \geq p_{0}>0, \quad \text { for any } 0<u<\infty \quad \text { if } \gamma=1 \text { in (1.6). }
\end{aligned}
$$


Here $p_{0}$ is a constant and

$$
\begin{gathered}
\underline{u}:=M^{-1}\left(\min _{\lambda \in[0,1]}\left[M\left(\min \left\{\tilde{u},(1-\lambda) \eta_{0}+\lambda \min _{x \in[0,1]} u_{0}(x)\right\}\right)-2 E_{0}^{1 / 2}(\lambda)\right]-1\right), \\
\bar{U}:=M^{-1}\left(\max _{\lambda \in[0,1]}\left[M\left(\max \left\{\tilde{U},(1-\lambda) \eta_{0}+\lambda \max _{x \in[0,1]} u_{0}(x)\right\}\right)+2 E_{0}^{1 / 2}(\lambda)\right]+1\right), \\
E_{0}(\lambda)=\left(1+2 \gamma^{2} / p_{0}\right) \int_{0}^{1}\left[E\left((1-\lambda) \eta_{0}+\lambda u_{0}(x),(1-\lambda) T_{0}+\lambda \theta_{0}(x)\right)\right. \\
\left.\quad+\lambda_{0}^{2}(x) / 2\right] d x+\gamma^{2} \eta_{0}^{2}, \\
M(u)=\int_{1}^{u} \mu(w) d w, \quad E(u, \theta)=\Psi(u, \theta)-\Psi\left(\eta_{0}, T_{0}\right)-\left(\theta-T_{0}\right) \Psi_{\theta}(u, \theta),
\end{gathered}
$$

and $\Psi(u, \theta)$ is the Helmholtz free energy function. By the same method as in [3] we can show that $u$ is a priori bounded, $\underline{u}<u(x, t)<\bar{U}$. We assume that there are exponents $q, r$ satisfying the following conditions:

$$
\begin{aligned}
& \text { if } 0 \leq r \leq 2 / 3, \text { then }(3 r+2) / 6<q ; \\
& \text { if } 2 / 3<r<3 \text {, then }(4 r+2) / 7<q ; \\
& \text { if } 3 \leq r \text {, then }(5 r+1) / 8<q
\end{aligned}
$$

and, concerning the growth of the temperature, we require that there be positive constants $\nu, N_{1}, N$, possibly depending on $\underline{u}$ and/or $\bar{U}$, such that for any $\underline{u} \leq u \leq \bar{U}$, $0 \leq \theta<\infty$

$$
\begin{aligned}
& 0 \leq e(u, 0), \quad \nu\left(1+\theta^{r}\right) \leq e_{\theta}(u, \theta) \leq N\left(1+\theta^{r}\right), \\
&-N_{1}\left(1+\theta^{r+1}\right) \leq p_{u}(u, \theta) \leq-N\left(1+\theta^{r+1}\right), \\
&\left|p_{\theta}(u, \theta)\right| \leq N\left(1+\theta^{r}\right), \\
& \nu\left(1+\theta^{q}\right) \leq k(u, \theta) \leq N\left(1+\theta^{q}\right), \\
&\left|k_{u}(u, \theta)\right|+\left|k_{u u}(u, \theta)\right| \leq N\left(1+\theta^{q}\right) .
\end{aligned}
$$

Without loss of generality, we assume that the initial velocity satisfies

$$
\int_{0}^{1} v_{0}(x) d x=0 \quad \text { if } \gamma=0 .
$$

For the initial data, we assume that for some $\alpha \in(0,1),\left(u_{0}(x), v_{0}(x), \theta_{0}(x)\right) \in H^{1+\alpha} \times$ $H^{2+\alpha} \times H^{2+\alpha}$ with $u_{0}(x)>0, \theta_{0}(x)>0$ for all $x \in[0,1]$, and the initial data are compatible with the boundary condition (1.6).

We shall use the familiar notation $H^{n+\alpha}, H_{T}^{n+\alpha}$, and $B_{T}^{n+\alpha}$ for nonnegative integer $n$ and $\alpha \in(0,1), T>0$, whose definitions are the same as those in [14]. In general and without danger of confusion we will use the same symbol to denote state functions as well as their values along a thermodynamic process, e.g., $p(u, \theta)$ and $p(u(x, t), \theta(x, t))$. $L^{p}, 1 \leq p \leq \infty, H^{1}=W^{1,2}, H_{0}^{1}=W_{0}^{1,2}$ denote the usual Sobolev spaces on $(0,1) ;\|\cdot\|_{B}$ denotes the norm in the space $B,\|\cdot\|:=\|\cdot\|_{L^{2}}$. Analogously, $\partial_{t}$ or $\frac{d}{d t}$ or a subscript $t$ and $\partial_{x}$ or a subscript $x$ denote the derivative with respect to $t$ and $x$ in the distribution 
sense, respectively. Letters $C, C_{i}$ will denote universal constants depending only on the initial data, but independent of $t$, any length of time.

We are now in a position to state our main theorem.

TheOREm 1.1. Under the assumptions (1.10)-(1.11), (1.13)-(1.14), (1.16)-(1.17), (1.22)-(1.30) and the above assumptions on the initial data, the problem (1.1)-(1.3), (1.5)-(1.6) admits a unique global solution $(u(t), v(t), \theta(t)) \in B_{T}^{1+\alpha} \times H_{T}^{2+\alpha} \times H_{T}^{2+\alpha}$ for any $T>0$ with $\underline{u}<u(x, t)<\bar{U}$ and $\theta(x, t)>0$ on $[0,1] \times[0, \infty)$. Moreover, there exist positive constants $C_{1}, C_{2}, C_{3}$ such that for all $t \geq C_{1}$, it follows that

$$
\|v(t)\|_{H^{1}}+\left\|\theta(t)-T_{0}\right\|_{H^{1}}+\left\|u(t)-\eta_{0}\right\|_{H^{1}} \leq C_{2} \exp \left(-C_{B_{3}} t\right)
$$

and as $t \rightarrow+\infty$, we have

$$
\begin{gathered}
\left\|u-\eta_{0}\right\|_{H^{1}} \rightarrow 0, \quad\|v\|_{H^{1}} \rightarrow 0, \quad\|v\|_{L^{x}} \rightarrow 0, \\
\left\|\theta_{x}\right\| \rightarrow 0, \quad\left\|\theta-T_{0}\right\|_{H^{1}} \rightarrow 0, \quad\left\|\theta-T_{0}\right\|_{L^{x}} \rightarrow 0, \\
\gamma\left(v^{2}(0, t)+v^{2}(1, t)\right) \rightarrow 0, \quad\|p(u, \theta)\|_{H^{1}} \rightarrow 0, \quad\|\sigma(u, \theta)\| \rightarrow 0
\end{gathered}
$$

where $\left(\eta_{0}, 0, T_{0}\right)$ is the unique solution to the corresponding stationary problem to the problem (1.1)-(1.3), (1.5)-(1.6).

Now let us recall the related results on nonlinear one-dimensional thermoviscoelasticity. For solid-like materials, Dafermos [1], Dafermos and Hsiao [2] considered the following boundary conditions (stress free and thermally insulated):

$$
\sigma(0, t)=\sigma(1, t)=0, \quad \tilde{q}(0, t)=\tilde{q}(1, t)=0, \quad t \geq 0,
$$

and established existence of global smooth solutions to the equations (1.1)-(1.3) by applying the Leray-Schauder fixed point theorem. The techniques in [1] work when only one end of the body is stress-free while the other is fixed. By the same method as in [1] with necessary modifications, Jiang [3] established the global existence of a smooth solution to the problem (1.1)-(1.3) and (1.5)-(1.6) with constitutive relations (1.10) and (1.11). It is well known that the large-time behaviour of the system (1.1)-(1.3) is of great interest since the pressure function $p(u, \theta)$ is not necessarily monotone in $u$. Unfortunately, the problem has been open until now. Hsiao and Luo [9] first considered a kind of solid-like material with the following constitutive relations:

$$
e=c_{0} \theta, \quad \sigma=-p(u, \theta)+\mu(u) v_{x}, \quad p(u, \theta)=f(u) \theta, \quad \tilde{q}=-k(u) \theta_{x} .
$$

and (1.11) where $c_{0}>0$ is a constant, $f(u)$ and $k(u)$ are twice continuously differentiable for $u>0$ such that

$$
\begin{array}{ll}
k(u)>0 & \text { for } u>0 \\
f(u) \geq 0 & \text { if } 0<u<\tilde{u} \\
f(u) \leq 0 & \text { if } \tilde{U}<u<+\infty
\end{array}
$$

for some fixed $0<\tilde{u}<\tilde{U}<+\infty$. It is shown that in [9] the solution to the problem (1.1)-(1.3), (1.5)-(1.6) may tend to a unique state exponentially fast, or may possess phase transition phenomena, corresponding to whether $f(u)$ is monotone or not. For the 
latter, the large-time behaviour of $u$ is described by a Young measure whose support is confined to the set of zeroes of $f(u)$. For the following constitutive relations,

$$
e=c_{0} \theta, \quad \sigma=-p(u, \theta)+\mu(u) v_{x}, \quad \tilde{q}=-k(u, \theta) \theta_{x}
$$

and (1.11) with $c_{0}$ being a constant, and for the following growth conditions imposed upon $k(u, \theta)$ : for any given positive constants $a$ and $A$ : $0<a \leq A<+\infty$, there exist positive constants $\nu$ and $N$, possibly depending on $a$ and/or $A$, such that

$$
\nu \leq k(u, \theta) \leq N, \quad\left|k_{u}(u, \theta)\right| \leq N, \quad\left|k_{u u}(u, \theta)\right| \leq N, \quad\left|k_{\theta}(u, \theta)\right| \leq N
$$

for any $(u, \theta) \in[a, A] \times(0,+\infty)$, Hsiao and Jian [8] investigated the large-time behaviour of smooth solutions to the problem (1.1)-(1.3), (1.5)-(1.6). With the different assumptions on the exponents $q$ and $r$, the author [21] established an asymptotic behaviour of a smooth solution to the problem (1.1)-(1.3), (1.5)-(1.6). Our assumptions on the exponents $q$ and $r$ in this paper include those in [21]; so the results in this paper improve those in [21]. Zheng and Shen [24] proved the global existence of smooth solutions provided that the initial data are sufficiently small, and Kim [13] established the existence of global weak solutions in $L^{1} \cap B V$. Recently, Racke and Zheng [22] investigated the global existence, uniqueness, and asymptotic behaviour of weak solutions to the model in shape memory alloys also with a stress-free boundary condition on at least one end of the rod. Shen, Zheng and Zhu [25] also studied the global existence, uniqueness, and asymptotic behaviour of a weak solution to the model in shape memory alloys with clamped and thermally insulated endpoints. When the material is an ideal gas or a real gas, there are many independent investigations both on the existence of global classical solutions of (1.1)-(1.3) (see [10]-[13], [16]-[19]) and on the asymptotic behaviour (see [6]-[7], [15], [17]-[21]), and the references cited therein. Figure 1.1 describes the ranges of the exponents $q$ and $r$ in [3], [8], [9] and in this paper in the $(r, q)$-plane.

There are two main difficulties to prove our results. The first arises from the higherorder nonlinearities of $\theta$ in the system (1.1)-(1.3). In order to overcome this one, we make full use of Lemma 2.4 and interpolation techniques to reduce the higher order of $\theta$. The second is that in order to study the large-time behaviour we have to establish the uniform estimates depending only on the initial data, but independent of any length of time.

The main contribution concerning the results in this paper is as follows:

(i) With the slightly different assumptions on the constitutive relations, only the global existence of classical solutions was obtained in [3], and the asymptotic behaviour of smooth large solutions was established in [9] only for the special case: $e(u, \theta)=c_{0} \theta$, $\sigma\left(u, \theta, v_{x}\right)=-p(u, \theta)+\mu(u) v_{x}, p(u, \theta)=f(u) \theta, \tilde{q}\left(u, \theta, \theta_{x}\right)=-k(u, \theta) \theta_{x}$ (see (1.40)(1.41)). For more general constitutive relations (see (1.10)-(1.11) and (1.12)-(1.29)) than those mentioned above in [9], we establish the results on global existence and asymptotic behaviour.

The main contributions concerning the methods in this paper are as follows: 


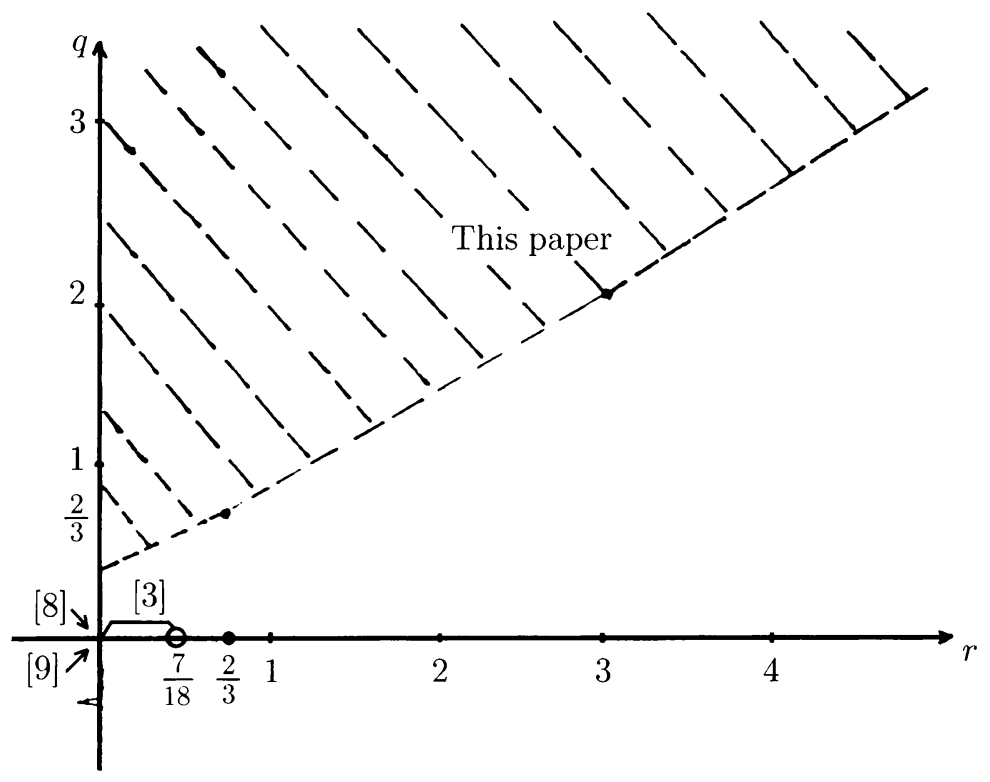

FIG. 1.1

(ii) We bound the norm of $u, v, \theta$ as well as their derivatives in terms of the expression of the form

$$
\left(1+\sup _{0 \leq s \leq t}\|\theta(s)\|_{L^{\infty}}\right)^{\Lambda}
$$

with $\Lambda$ being a positive constant depending only on the exponents $q$ and $r$.

(iii) We make full use of Lemma 2.4 and more delicate interpolation techniques to reduce the inherent higher order of $\theta$ in the system considered.

(iv) A new method is introduced to show the asymptotic behaviour of the solutions (see Lemma 3.1-Lemma 3.4) by first establishing a function $Z(t)$ (see (3.9)) related to the norm $\|(u, v, \theta)\|_{H^{1}}$ of the solutions $(u, v, \theta)$ and then applying a useful basic lemma (see Lemma 3.1) in analysis by Shen and Zheng in [23] to $Z(t)$.

The aims of the present paper are

(i) to establish the global existence, uniqueness, and asymptotic behaviour of solutions;

(ii) to investigate how the exponents $q$ and $r$ affect the global existence and asymptotic behaviour of solutions.

We organize our present paper as follows. In Sec. 2, we shall first get uniform a priori estimates of the solution. In Sec. 3, we shall deduce the results of asymptotic behaviour by applying a basic lemma (Lemma 3.1) in analysis by Shen and Zheng [23].

REMARK 1.1. With the different assumptions on the exponents $q$ and $r$, the similar conclusions to those in Theorem 1.1 hold for the boundary conditions

$$
\sigma(0, t)=v(0, t), \quad \sigma(1, t)=-v(1, t), \quad \tilde{q}(0, t)=\tilde{q}(1, t)=0
$$


and for the boundary conditions

$$
\begin{gathered}
\tilde{q}(0, t)=\tilde{q}(1, t)=0 \quad \text { or } \quad \theta(0, t)=\theta(1, t)=T_{0}, \\
\sigma(0, t)=0, \sigma(1, t)=-v(1, t) \quad \text { or } \quad \sigma(0, t)=v(0, t), \sigma(1, t)=0 .
\end{gathered}
$$

REMARK 1.2. The similar results of global existence in Theorem 1.1 can be established for the boundary conditions $\sigma(0, t)=\sigma(1, t)=0, \tilde{q}(0, t)=\tilde{q}(1, t)=0$, and $\sigma(0, t)=$ $\sigma(1, t)=0, \theta(0, t)=\theta(1, t)=T_{0}$. With the exponents $q=0$ and $0 \leq r<7 / 18$, Dafermos [1] established the global existence of smooth solutions for the first case of boundary conditions above.

2. Uniform a priori estimates. The existence and uniqueness of local solutions (with positive $u$ and $\theta$ ) can be obtained by linearization of the problem (1.1)-(1.3), (1.5)(1.6), and by use of the Banach contraction mapping theorem. The global existence of solutions in Theorem 1.1 is based on a priori estimates that can be used to continue a local solution globally in time.

Theorem 2.1. Let $(u, v, \theta)$ be a smooth solution as described in Theorem 1.1. Then we have for any $T>0$,

$$
\|\| u\left\|_{T}^{(1+\alpha)}+\right\| v\left\|_{T}^{(2+\alpha)}+\right\|\|\theta\|_{T}^{(2+\alpha)} \leq C .
$$

The proofs of Theorem 2.1 and Theorem 1.1 are divided into a series of lemmas.

LEMMA 2.1.

$$
\begin{aligned}
\theta(x, t) & >0, & & \text { on }[0,1] \times[0, \infty), \\
\underline{u}<u(x, t) & <\bar{U}, & & \text { on }[0,1] \times[0, \infty)
\end{aligned}
$$

where $\underline{u}$ and $\bar{U}$ are defined by (1.18) and (1.19), respectively.

Proof. See [3].

\section{LEMMA 2.2 .}

$$
\begin{aligned}
\int_{0}^{1}\left[\left(\theta / T_{0}-\log \left(\theta / T_{0}\right)-1\right)+\right. & \left.\theta^{1+r}+v^{2}\right] d x+\gamma \int_{0}^{t}\left[v^{2}(0, s)+v^{2}(1, s)\right] d s \\
& +\int_{0}^{t} \int_{0}^{1}\left[\frac{v_{x}^{2}}{\theta}+\frac{\left(1+\theta^{q}\right) \theta_{x}^{2}}{\theta^{2}}\right] d x d s \leq C_{4}, \quad \forall t>0 .
\end{aligned}
$$

Proof. Since $\Psi(u, \theta)=e(u, \theta)-\theta \eta(u, \theta)$ satisfies

$$
-\Psi_{\theta}(u, \theta)=\eta(u, \theta), \quad \Psi_{u}(u, \theta)=\sigma(u, \theta, 0)=-p(u, \theta),
$$

thus, by (1.1)-(1.3), (1.10) and (2.4), and noting that $e_{\theta}(u, \theta)=-\theta \Psi_{\theta \theta}(u, \theta)$, we deduce after a direct calculation that

$$
\partial_{t}\left[E(u, \theta)+\frac{v^{2}}{2}\right]+T_{0}\left[\frac{\mu v_{x}^{2}}{\theta}+\frac{k(u, \theta) \theta_{x}^{2}}{\theta^{2}}\right]=\left[\sigma v+\frac{\left(\theta-T_{0}\right) k(u, \theta) \theta_{x}}{\theta}\right]_{x} .
$$


Integrating (2.5) over $Q_{t}$ and using (1.6) lead to

$$
\begin{aligned}
\int_{0}^{1}\left(E(u, \theta)+\frac{v^{2}}{2}\right)( & x, t) d x+T_{0} \int_{0}^{t} \int_{0}^{1}\left(\frac{\mu v_{x}^{2}}{\theta}+\frac{k(u, \theta) \theta_{x}^{2}}{\theta^{2}}\right) d x d s \\
& +\gamma \int_{0}^{t}\left[v^{2}(0, s)+v^{2}(1, s)\right] d s=\int_{0}^{1}\left(E\left(u_{0}, \theta_{0}\right)+\frac{v_{0}^{2}}{2}\right) d x
\end{aligned}
$$

In view of $(1.16)-(1.17)$, we have $\Psi_{u u}\left(u, T_{0}\right)=-p_{u}\left(u, T_{0}\right)>0$ for $u \in[\underline{u}, \bar{U}]$. Therefore, it follows from the Taylor theorem and (1.21) that

$$
\begin{aligned}
& E(u, \theta)-\Psi(u, \theta)+\Psi\left(u, T_{0}\right)+\left(\theta-T_{0}\right) \Psi_{\theta}(u, \theta) \\
& \quad=\Psi\left(u, T_{0}\right)-\Psi\left(\eta_{0}, T_{0}\right)-\Psi_{u}\left(\eta_{0}, T_{0}\right)\left(u-\eta_{0}\right) \\
& =\left(u-\eta_{0}\right)^{2} \int_{0}^{1}(1-\xi) \Psi_{u u}\left(\eta_{0}+\xi\left(u-\eta_{0}\right), T_{0}\right) d \xi \geq 0 .
\end{aligned}
$$

Thus,

$$
\begin{aligned}
& E(u, \theta) \geq \Psi(u, \theta)-\Psi\left(u, T_{0}\right)-\left(\theta-T_{0}\right) \Psi_{\theta}(u, \theta) \\
& =-\left(T_{0}-\theta\right)^{2} \int_{0}^{1}(1-\tau) \Psi_{\theta \theta}\left(u, \theta+\tau\left(T_{0}-\theta\right)\right) d \tau \\
& \geq \nu\left(T_{0}-\theta\right)^{2} \int_{0}^{1} \frac{(1-\tau)\left\{1+\left[\theta+\tau\left(T_{0}-\theta\right)\right]^{r}\right\}}{\theta+\tau\left(T_{0}-\theta\right)} d \tau \\
& =\left\{\begin{array}{l}
2 \nu T_{0}\left(\theta / T_{0}-\log \left(\theta / T_{0}\right)-1\right) \text { if } r=0 \\
\nu T_{0}\left(\theta / T_{0}-\log \left(\theta / T_{0}\right)-1\right)+\frac{\nu^{\prime} T_{0}\left(T_{0}^{\prime \prime}-\theta^{\prime \prime}\right)}{r}-\frac{\nu\left(T_{0}^{1+r}-\theta^{1+r}\right)}{r+1} \text { if } r \neq 0
\end{array}\right. \\
& \geq \nu T_{0}\left(\theta / T_{0}-\log \left(\theta / T_{0}\right)-1\right)+C_{5} \theta^{r+1}-C_{6}
\end{aligned}
$$

which, combined with (1.26) and (2.6), yields (2.3).

REMARK 2.1. It follows from the convexity of the function $-\ln y$ that there exist two positive constants $r_{1}, r_{2}$ depending only on the initial data such that

$$
0<r_{1} \leq \frac{1}{T_{0}} \int_{0}^{1} \theta d x \leq r_{2} .
$$

REMARK 2.2. By the mean-value theorem and (1.15), we have

$$
|p(u, \theta)| \leq C\left(1+\theta^{r+1}\right)
$$

for any $u \in[\underline{u}, \bar{U}]$ and $\theta>0$.

Hereafter, we set

$$
B(t)=1+\sup _{0 \leq s \leq t}\|\theta(s)\|_{L^{x}}
$$

LEMMA 2.3 .

$$
\begin{gathered}
\int_{0}^{t}\|v\|_{L^{x}}^{2} d s \leq C, \quad \forall t>0, \\
\int_{0}^{t}\left\|v_{x}\right\|^{2} d s \leq C B(t)^{\beta}, \quad \forall t>0
\end{gathered}
$$

with $\beta=\max (r+1-q, 0)$. 
Proof. By the same method as in [3] or [9], we can easily prove (2.8). Multiplying (1.2) by $v$, integrating the resultant over $Q_{t}$ and using (1.1), (1.6), (1.11), (1.24), Lemma 2.1 , the mean-value theorem, and Remark 2.2 , yield

$$
\begin{aligned}
\frac{1}{2}\|v\|^{2}+ & \gamma \int_{0}^{t}\left(v^{2}(0, s)+v^{2}(1, s)\right) d s+\int_{0}^{t} \int_{0}^{1} \mu(u) v_{x}^{2} d x d s \\
= & \int_{0}^{t} \int_{0}^{1}\left(p(u, \theta)-p\left(u, T_{0}\right)\right) v_{x} d x d s+\int_{0}^{t} \int_{0}^{1} p\left(u, T_{0}\right) v_{x} d x d s+\frac{1}{2}\left\|v_{0}\right\|^{2} \\
\leq & C \int_{0}^{t}\left\|p_{\theta}\left(u, \theta_{2}\right)\left(\theta-T_{0}\right)\right\|^{2} d s+\frac{1}{2} \int_{0}^{t} \int_{0}^{1} \mu(u) v_{x}^{2} d x d s \\
& +\left|\int_{0}^{1} \int_{\underline{u}}^{u} p\left(\xi, T_{0}\right) d \xi d x\right|+\left|\int_{0}^{1} \int_{\underline{u}}^{u_{0}} p\left(\xi, T_{0}\right) d \xi d x\right|+C \\
\leq & C+\frac{1}{2} \int_{0}^{t} \int_{0}^{1} \mu(u) v_{x}^{2} d x d s+C \int_{0}^{t}\left\|(1+\theta)^{(r-1) / 2}\left(\theta-T_{0}\right)\right\|_{L^{\infty}}^{2} \int_{0}^{1}(1+\theta)^{r+1} d x d s \\
\leq & C+\frac{1}{2} \int_{0}^{t} \int_{0}^{1} \mu(u) v_{x}^{2} d x d s+C \int_{0}^{t}\left\|\left[(1+\theta)^{(r-1) / 2}\left(\theta-T_{0}\right)\right]_{x}\right\|_{L^{1}}^{2} d s \\
\leq & C+\frac{1}{2} \int_{0}^{t} \int_{0}^{1} \mu(u) v_{x}^{2} d x d s+C B(t)^{\beta} \int_{0}^{t} \int_{0}^{1} \frac{\left(1+\theta^{q}\right) \theta_{x}^{2}}{\theta^{2}} d x d s \\
\leq & C B(t)^{\beta}+\frac{1}{2} \int_{0}^{t} \int_{0}^{1} \mu(u) v_{x}^{2} d x d s
\end{aligned}
$$

which gives (2.9); here $\min \left(\theta, T_{0}\right) \leq \theta_{2} \leq \max \left(\theta, T_{0}\right) \leq C(1+\theta)$.

LEMMA 2.4 .

$$
C-C V(t) \leq \theta^{2 m_{1}}(x, t) \leq C+C V(t), \quad \forall(x, t) \in[0,1] \times[0,+\infty)
$$

with $0 \leq m_{1} \leq m=(q+r+1) / 2$ and $V(t)=\int_{0}^{1} \frac{\left(1+\theta^{q}\right) \theta_{x}^{2}}{\theta^{2}} d x$ satisfying $\int_{0}^{\infty} V(t) d t<\infty$.

Proof. The Cauchy inequality, Lemma 2.1 and (1.6) imply

$$
\begin{aligned}
\theta^{m_{1}} & \leq C+C \int_{0}^{1}\left|\theta^{m_{1}-1} \theta_{x}\right| d x \\
& \leq C+C V^{1 / 2}(t)
\end{aligned}
$$

which implies (2.10).

LEMMA 2.5.

$$
\begin{gathered}
\int_{0}^{t} \int_{0}^{1}(1+\theta)^{2 m} v^{2} d x d s \leq C, \quad \forall t>0, \\
\left\|u_{x}\right\|^{2}+\int_{0}^{t} \int_{0}^{1}\left(1+\theta^{r+1}\right) u_{x}^{2} d x d s \leq C B(t)^{\beta}, \quad \forall t>0, \\
\int_{0}^{t} \int_{0}^{1}(1+\theta)^{2 m} u_{x}^{2} d x d s \leq C B(t)^{\beta}, \quad \forall t>0 .
\end{gathered}
$$


Proof. It follows from Lemma 2.1 and Lemmas 2.3-2.4 that

$$
\int_{0}^{t} \int_{0}^{1}(1+\theta)^{2 m} v^{2} d x d s \leq C \int_{0}^{t} \int_{0}^{1} v^{2} d x d s+C \int_{0}^{t} V(s)\|v\|^{2} d s \leq C .
$$

Equation (1.2) can be rewritten as

$$
\left(v-\mu(u) u_{x}\right)_{t}+p_{u}(u, \theta) u_{x}=-p_{\theta}(u, \theta) \theta_{x}
$$

Multiplying (2.14) by $v-\mu(u) u_{x}$, then integrating the resultant over $Q_{t}$, using Lemmas 2.1-2.4 and noting the following facts

$$
\begin{aligned}
\int_{0}^{t} \int_{0}^{1} \frac{\theta^{2}\left(1+\theta^{r}\right)^{2} v^{2}}{1+\theta^{q}} d x d s & \leq C B(t)^{\delta} \int_{0}^{t} \int_{0}^{1}(1+\theta)^{2 m} v^{2} d x d s \\
& \leq C B(t)^{\delta} \\
\int_{0}^{t} \int_{0}^{1} \frac{\left(1+\theta^{r}\right)^{2} \theta_{x}^{2}}{1+\theta^{r+1}} d x d s & \leq C \int_{0}^{t} \int_{0}^{1}(1+\theta)^{r-1} \theta_{x}^{2} d x d s \\
& \leq C B(t)^{\beta} \int_{0}^{t} V(s) d s \leq C B(t)^{\beta}
\end{aligned}
$$

we arrive at

$$
\begin{aligned}
\left\|u_{x}\right\|^{2} & +\int_{0}^{t} \int_{0}^{1}\left[1+\theta^{r+1}\right] u_{x}^{2} d x d s \\
\leq & C+C \int_{0}^{t} \int_{0}^{1}\left[\left(1+\theta^{r+1}\right)\left|u_{x} v\right|+\left(1+\theta^{r}\right) \mid \theta_{x}\left(v-\mu(u) u_{x} \mid\right] d x d s\right. \\
\leq & C+C \int_{0}^{t} \int_{0}^{1}\left(1+\theta^{1+r}\right)\left(\varepsilon u_{x}^{2}+C v^{2}\right) d x d s+C \int_{0}^{t} V(s) d s \\
& +C \int_{0}^{t} \int_{0}^{1} \frac{\theta^{2}\left(1+\theta^{r}\right)^{2}}{1+\theta^{q}} v^{2} d x d s+C \varepsilon \int_{0}^{t} \int_{0}^{1}\left(1+\theta^{1+r}\right) u_{x}^{2} d x d s \\
& +C \int_{0}^{t} \int_{0}^{1} \frac{\left(1+\theta^{r}\right)^{2} \theta_{x}^{2}}{1+\theta^{1+r}} d x d s \\
\leq & C B(t)^{\beta}+C \varepsilon \int_{0}^{t} \int_{0}^{1}\left(1+\theta^{1+r}\right) u_{x}^{2} d x d s+C \int_{0}^{t}\|v\|_{L^{\infty}}^{2} d s
\end{aligned}
$$

with $\beta=\max (r+1-q, 0) \geq \delta=\max (r+1-2 q, 0)$. Thus, taking $\varepsilon>0$ small enough in (2.15) yields (2.12). The proof of (2.13) is similar to that of (2.11) if we exploit (2.12).

LEMMA 2.6.

$$
\begin{array}{r}
\left\|v_{x}\right\|^{2}+\int_{0}^{t} \int_{0}^{1} v_{t}^{2} d x d s+\gamma\left(v^{2}(0, t)+v^{2}(1, t)\right) \leq C B(t)^{\beta_{4}}, \quad \forall t>0 \\
\int_{0}^{t}\left\|v_{x x}\right\|^{2} d s \leq C B(t)^{\beta_{5}}, \quad \forall t>0
\end{array}
$$


with

$$
\begin{aligned}
& \beta_{1}=\max (2 r+2-q, 0), \\
& \beta_{2}=\max \left(3 \beta, \beta_{1}\right), \\
& \beta_{3}=\left(\beta_{2}+3 \beta\right) / 2, \\
& \beta_{4}=\max \left(\beta_{1}, \beta_{3}, 3 \beta\right), \\
& \beta_{5}=\max \left(\beta_{2}, \beta_{4}\right) .
\end{aligned}
$$

Proof. By (1.2), we have

$$
v_{x x}=\mu^{-1}(u)\left(v_{t}+p_{u} u_{x}+p_{\theta} \theta_{x}-\mu^{\prime}(u) u_{x} v_{x}\right)
$$

It follows from Lemmas $2.1-2.6$ that

$$
\begin{aligned}
\int_{0}^{t}\left\|v_{x x}\right\|^{2} d s \\
\leq C \int_{0}^{t}\left(\left\|v_{t}\right\|^{2}+\left\|\left(1+\theta^{r+1}\right) u_{x}\right\|^{2}+\left\|\left(1+\theta^{r}\right) \theta_{x}\right\|^{2}+\left\|v_{x}\right\|_{L^{\infty}}^{2}\left\|u_{x}\right\|^{2}\right) d s \\
\leq C B(t)^{\beta} \int_{0}^{t} \int_{0}^{1}(1+\theta)^{2 m} u_{x}^{2} d x d s+C \int_{0}^{t}\left\|v_{t}\right\|^{2} d s \\
\quad+C B(t)^{\beta_{1}} \int_{0}^{t} V(s) d s+C B(t)^{\beta} \int_{0}^{t}\left(\left\|v_{x}\right\|\left\|v_{x x}\right\|+\left\|v_{x}\right\|^{2}\right) d s \\
\leq C B(t)^{\beta_{1}}+C B(t)^{3 \beta}+C \int_{0}^{t}\left\|v_{t}\right\|^{2} d s+\frac{1}{2} \int_{0}^{t}\left\|v_{x x}\right\|^{2} d s
\end{aligned}
$$

which implies

$$
\int_{0}^{t}\left\|v_{x x}\right\|^{2} d s \leq C B(t)^{\beta_{2}}+C \int_{0}^{t}\left\|v_{t}\right\|^{2} d s
$$

By virtue of (1.1) and (1.6), we easily get

$$
\begin{gathered}
\left.\mu(u) v_{x} v_{t}\right|_{x=1} \\
=-\frac{\gamma}{2} \frac{d}{d t} v^{2}(1, t)+\frac{d}{d t}\left(p\left(u(1, t), T_{0}\right) v(1, t)\right) \\
\quad-p_{u}\left(u(1, t), T_{0}\right) v_{x}(1, t) v(1, t), \\
\left.\mu(u) v_{x} v_{t}\right|_{x=0} \\
=\frac{\gamma}{2} \frac{d}{d t} v^{2}(0, t)+\frac{d}{d t}\left(p\left(u(0,1), T_{0}\right) v(0, t)\right) \\
\quad-p_{u}\left(u(0, t), T_{0}\right) v_{x}(0, t) v(0, t) .
\end{gathered}
$$


On the other hand, multiplying (1.2) by $v_{t}$, then integrating the resultant over $Q_{t}$, using the Nirenberg inequality, (2.18)-(2.20) and Lemmas 2.1-2.6, yield

$$
\begin{aligned}
& \left\|v_{x}\right\|^{2}+\int_{0}^{t}\left\|v_{t}\right\|^{2} d s+\gamma\left(v^{2}(0, t)+v^{2}(1, t)\right) \\
& \leq C+\varepsilon \int_{0}^{t}\left\|v_{t}\right\|^{2} d s+C \int_{0}^{t}\left(\left\|v_{x}\right\|_{L^{3}}^{3}+\left\|p_{u} u_{x}+p_{\theta} \theta_{x}\right\|^{2}\right) d s \\
& +C\left|p\left(u(1, t), T_{0}\right) v(1, t)-p\left(u(0, t), T_{0}\right) v(0, t)\right| \\
& +C \int_{0}^{t}\left(\left|p_{u}\left(u(1, t), T_{0}\right) v_{x}(1, t) v(1, t)\right|+\left|p_{u}\left(u(0, t), T_{0}\right) v_{x}(0, t) v(0, t)\right|\right) d s \\
& \leq \varepsilon \int_{0}^{t}\left\|v_{t}\right\|^{2} d s+C \int_{0}^{t}\left(\left\|v_{x}\right\|^{5 / 2}\left\|v_{x x}\right\|^{1 / 2}+\left\|v_{x}\right\|^{3}\right) d s+C\|v\|_{L^{\infty}} \\
& +C B(t)^{2 \beta}+C B(t)^{\beta_{1}}+C\left(\int_{0}^{t}\|v\|_{L^{x}}^{2} d s\right)^{1 / 2}\left(\int_{0}^{t}\left\|v_{x}\right\|_{L^{\infty}}^{2} d s\right)^{1 / 2} \\
& \leq C B(t)^{\beta_{1}}+\varepsilon \int_{0}^{t}\left\|v_{t}\right\|^{2} d s+C\left\|v_{x}\right\|^{1 / 2} \\
& +C\left(\int_{0}^{t}\left\|v_{x x}\right\|^{2} d s\right)^{1 / 4}\left(\int_{0}^{t}\left\|v_{x}\right\|^{2} d s\right)^{3 / 4} \sup _{0 \leq s \leq t}\left\|v_{x}\right\|+C \sup _{0 \leq s \leq t}\left\|v_{x}\right\| \int_{0}^{t}\left\|v_{x}\right\|^{2} d s \\
& +C\left(\int_{0}^{t}\left\|v_{x}\right\|^{2} d s\right)^{1 / 2}+C\left(\int_{0}^{t}\left\|v_{x}\right\|^{2} d s\right)^{1 / 4}\left(\int_{0}^{t}\left\|v_{x x}\right\|^{2} d s\right)^{1 / 4} \\
& \leq C B(t)^{\beta_{1}}+C B(t)^{\beta_{3} / 2} \sup _{0 \leq s \leq t}\left\|v_{x}\right\|+\varepsilon \sup _{0 \leq s \leq t}\left\|v_{x}\right\|^{2}+\varepsilon \int_{0}^{t}\left\|v_{t}\right\|^{2} d s \\
& +C\left(\int_{0}^{t}\left\|v_{t}\right\|^{2} d s\right)^{1 / 4} B(t)^{3 \beta / 4} \sup _{0 \leq s \leq t}\left\|v_{x}\right\|+C \sup _{0 \leq s \leq t}\left\|v_{x}\right\| B(t)^{\beta} \\
& +C B(t)^{\left(\beta+\beta_{2}\right) / 4}+C B(t)^{\beta / 4}\left(\int_{0}^{t}\left\|v_{t}\right\|^{2} d s\right)^{1 / 4}+C B(t)^{\beta / 2} \\
& \leq C B(t)^{\beta_{1}}+2 \varepsilon \sup _{0 \leq s \leq t}\left\|v_{x}\right\|^{2}+2 \varepsilon \int_{0}^{t}\left\|v_{t}\right\|^{2} d s+C B(t)^{\beta_{3}}+C B(t)^{3 \beta} \\
& \leq C B(t)^{\beta_{4}}+2 \varepsilon \sup _{0 \leq s \leq t}\left\|v_{x}\right\|^{2}+2 \varepsilon \int_{0}^{t}\left\|v_{t}\right\|^{2} d s
\end{aligned}
$$

which gives (2.16) for small $\varepsilon>0$, and (2.17) follows from (2.16) and (2.18).

Corollary 2.1 .

$$
\begin{gathered}
\int_{0}^{t}\left\|p_{x}\right\|^{2} d s \leq C B(t)^{\beta_{1}}, \quad \forall t>0 \\
\int_{0}^{t}\left\|v_{x}\right\|_{L^{3}}^{3} d s \leq C B(t)^{\beta_{6}}, \quad \forall t>0 \\
\int_{0}^{t}\left\|v_{x}\right\|_{L^{\infty}}^{2} d s \leq C B(t)^{\beta_{7}}, \quad \forall t>0
\end{gathered}
$$


with $\beta_{6}=\left(\beta_{5}+3 \beta+2 \beta_{4}\right) / 4$ and $\beta_{7}=\left(\beta+\beta_{5}\right) / 2$.

Proof. By Lemmas 2.1-2.7, we have

$$
\begin{gathered}
\int_{0}^{t}\left\|p_{x}\right\|^{2} d s \leq C \int_{0}^{t} \int_{0}^{1}\left[(1+\theta)^{2 r+2} u_{x}^{2}+(1+\theta)^{2 r} \theta_{x}^{2}\right] d x d s \\
\leq C B(t)^{2 \beta}+C B(t)^{\beta_{1}} \leq C B(t)^{\beta_{1}}, \\
\int_{0}^{t}\left\|v_{x}\right\|_{L^{3}}^{3} d s \leq C \int_{0}^{t}\left[\left\|v_{x}\right\|^{5 / 2}\left\|v_{x x}\right\|^{1 / 2}+\left\|v_{x}\right\|^{3}\right] d s \\
\leq C\left[\left(\int_{0}^{t}\left\|v_{x x}\right\|^{2} d s\right)^{1 / 4}\left(\int_{0}^{t}\left\|v_{x}\right\|^{2} d s\right)^{3 / 4}+\int_{0}^{t}\left\|v_{x}\right\|^{2} d s\right] \sup _{0 \leq s \leq t}\left\|v_{x}\right\| \\
\leq C B(t)^{\left(\beta_{5}+3 \beta+2 \beta_{4}\right) / 4}+C B(t)^{\beta_{4} / 2+\beta} \leq C B(t)^{\beta_{6}},
\end{gathered}
$$

and

$$
\begin{aligned}
\int_{0}^{t}\left\|v_{x}\right\|_{L^{x}}^{2} d s & \leq C \int_{0}^{t}\left[\left\|v_{x}\right\|\left\|v_{x x}\right\|+\left\|v_{x}\right\|^{2}\right] d s \\
& \leq C\left(\int_{0}^{t}\left\|v_{x}\right\|^{2} d s\right)^{1 / 2}\left(\int_{0}^{t}\left\|v_{x x}\right\|^{2} d s\right)^{1 / 2}+C \int_{0}^{t}\left\|v_{x}\right\|^{2} d s \\
& \leq C B(t)^{\left(\beta+\beta_{5}\right) / 2}+C B(t)^{\beta} \leq C B(t)^{\beta_{7}}
\end{aligned}
$$

with $\beta_{5} \geq 3 \beta$.

LEMMA 2.7 .

$$
\begin{aligned}
&\|v\|_{H^{1}} \leq C B(t)^{\beta_{4} / 2}, \quad \forall t>0,\|v\|_{L^{\infty}} \leq C B(t)^{\beta_{4} / 4}, \quad \forall t>0, \\
& \int_{0}^{t} \int_{0}^{1}(1+\theta)^{2 m} v_{x}^{2} d x d s \leq C B(t)^{\beta_{4}}, \quad \forall t>0, \\
& \int_{0}^{t} \int_{0}^{1}(1+\theta)^{2 m} v^{2} v_{x}^{2} d x d s \leq C B(t)^{3 \beta_{4} / 2}, \quad \forall t>0, \\
&\|v\|_{L^{4}}^{4}+\gamma \int_{0}^{t}\left(v^{4}(0, s)+v^{4}(1, s)\right) d s+\int_{0}^{t}\left\|v v_{x}\right\|^{2} d s \leq C B(t)^{\beta}, \quad \forall t>0 .
\end{aligned}
$$

Proof. From the Nirenberg inequality, Lemma 2.5, and Lemma 2.7, we easily deduce (2.24)-(2.26). Multiplying (1.2) by $v^{3}$, integrating the resultant over $Q_{t}$, and using (1.6) and Lemma 2.6, give

$$
\begin{aligned}
\|v\|_{L^{4}}^{4} & +\gamma \int_{0}^{t}\left(v^{4}(0, s)+v^{4}(1, s)\right) d s+\int_{0}^{t} \int_{0}^{1} v^{2} v_{x}^{2} d x d s \\
& \leq C+C \int_{0}^{t} \int_{0}^{1}(1+\theta)^{r+1} v^{2}\left|v_{x}\right| d x d s \\
& \leq C B(t)^{\beta}+\frac{1}{2} \int_{0}^{t} \int_{0}^{1} v^{2} v_{x}^{2} d x d s
\end{aligned}
$$


which implies (2.28), and (2.27) is the direct result of Lemma 2.4, (2.24)-(2.25), and (2.28).

\section{LEMMA 2.8 .}

$$
\left\|\theta^{1+r}\right\|^{2}+\int_{0}^{t} \int_{0}^{1}\left[\frac{\left(T_{0}-\theta\right)^{2}(1+\theta)^{q+r} \theta_{x}^{2}}{\theta^{2}}+(1+\theta)^{q+r-1} \theta_{x}^{2}\right] d x d s \leq C B(t)^{\beta_{8}}, \quad \forall t>0
$$

with

$$
\begin{aligned}
\beta_{9} & =\min [\max (2 r+1-2 q, 0), \max (3 r+3-2 q, 0) / 2], \\
\beta_{10} & =\min \left[\max (r-q, 0)+\beta, \delta / 2+3 \beta_{4} / 4,\left(\beta+\beta_{1}\right) / 2\right], \\
\beta_{11} & =\min \left[\max (q-r, 0)+\beta,(q+2+\beta) / 2,\left(2 \max (1-r, 0)+3 \beta_{4}\right) / 4\right], \\
\beta_{12} & =\beta+1 \\
\beta_{8} & =\max \left(3 \beta / 2, \beta_{9}, \beta_{10}, \beta_{11}, \beta_{12}\right) .
\end{aligned}
$$

Proof. Let

$$
E_{1}(u, \theta)=E(u, \theta)+C_{6}, A(t)=\int_{0}^{t} \int_{0}^{1} \frac{\left(T_{0}-\theta\right)^{2}(1+\theta)^{q+r} \theta_{x}^{2}}{\theta^{2}} d x d s .
$$

Thus we know from the proof of Lemma 2.2 that

$$
E_{1}(u, \theta) \geq C_{5} \theta^{r+1}>0
$$

Multiplying (2.5) by $E_{1}+\frac{v^{2}}{2}$, integrating the resultant over $Q_{t}$ yield

$$
\begin{aligned}
& \left\|E_{1}(u, \theta)+\frac{v^{2}}{2}\right\|^{2}+2 T_{0} \int_{0}^{t} \int_{0}^{1}\left[\frac{\mu v_{x}^{2}}{\theta}+\frac{k(u, \theta) \theta_{x}^{2}}{\theta^{2}}\right]\left(E_{1}(u, \theta)+v^{2} / 2\right) d x d s \\
& +2 \gamma \int_{0}^{t}\left[v^{2}(0, s)\left(E_{1}(0, s)+\frac{v^{2}(0, s)}{2}\right)+v^{2}(1, s)\left(E_{1}(1, s)+\frac{v^{2}(1, s)}{2}\right)\right] d s \\
& =\left\|E_{1}\left(u_{0}, \theta_{0}\right)+\frac{v_{0}^{2}}{2}\right\|^{2}-2 \int_{0}^{t} \int_{0}^{1}\left(\sigma v+\frac{\left(\theta-T_{0}\right) k(u, \theta) \theta_{x}}{\theta}\right)\left(E_{x}+v v_{x}\right) d x d s
\end{aligned}
$$

Noting (2.4) and (1.21), we can deduce

$$
E_{x}(u, \theta)=-p(u, \theta) u_{x}+p_{\theta}(u, \theta)\left(\theta-T_{0}\right) u_{x}+e_{\theta}(u, \theta) \frac{\left(\theta-T_{0}\right) \theta_{x}}{\theta} .
$$

Inserting (2.31) and (1.10)-(1.11) into (2.30), and using Lemma 2.8 and Corollary 2.1, lead to

$$
\begin{aligned}
& \left\|\theta^{r+1}\right\|^{2}+\int_{0}^{t} \int_{0}^{1}\left[\left(\theta^{r-1}+\theta^{q+r-1}\right) \theta_{x}^{2}+\left(T_{0}-\theta\right)^{2}(1+\theta)^{q+r} \theta_{x}^{2} \theta^{-2}\right] d x d s \\
& \leq C+I_{1}+I_{2}+I_{3}+I_{4} .
\end{aligned}
$$


By Lemmas 2.1-2.8, we have

$$
\begin{aligned}
I_{1} \leq C \int_{0}^{t} \int_{0}^{1}\left[(1+\theta)^{2 r+2}\left|v u_{x}\right|+(1+\theta)^{2 r+1}\left|\left(T_{0}-\theta\right) \theta_{x} v\right| \theta^{-1}\right. \\
\left.+(1+\theta)^{r+1} v^{2}\left|v_{x}\right|\right] d x d s \\
=I_{1}^{(1)}+I_{1}^{(2)}+I_{1}^{(3)}
\end{aligned}
$$

where

$$
\begin{gathered}
I_{1}^{(1)} \leq C\left(\int_{0}^{t} \int_{0}^{1}(1+\theta)^{2 r+2} u_{x}^{2} d x d s\right)^{1 / 2}\left(\int_{0}^{t} \int_{0}^{1}(1+\theta)^{2 r+2} v^{2} d x d s\right)^{1 / 2} \\
\leq C B(t)^{3 \beta / 2} \\
I_{1}^{(2)} \leq C \int_{0}^{t} \int_{0}^{1} \frac{(1+\theta)^{2 r+1}\left|\left(T_{0}-\theta\right) v \theta_{x}\right|}{\theta} d x d s \\
\leq \frac{1}{8} A(t)+C B(t)^{\max (2 r+1-2 q, 0)}
\end{gathered}
$$

or

$$
\begin{aligned}
I_{1}^{(2)} & \leq C\left(\int_{0}^{t} V(s) d s\right)^{1 / 2}\left(\int_{0}^{t} \int_{0}^{1}(1+\theta)^{4 r+4-q} v^{2} d x d s\right)^{1 / 2} \\
& \leq C B(t)^{\max (3 r+3-2 q, 0) / 2}
\end{aligned}
$$

which, combined with (2.35), yields

$$
I_{1}^{(2)} \leq \frac{1}{8} A(t)+C B(t)^{\beta_{9}} .
$$

Similarly,

$$
\begin{aligned}
I_{1}^{(3)} & \leq C \int_{0}^{t} \int_{0}^{1}(1+\theta)^{r+1} v^{2}\left|v_{x}\right| d x d s \\
& \leq C B(t)^{\beta}
\end{aligned}
$$

and

$$
\begin{aligned}
I_{2} & \leq C \int_{0}^{t} \int_{0}^{1}\left[(1+\theta)^{r+1}\left|u_{x} v v_{x}\right|+\frac{(1+\theta)^{r}\left|\left(T_{0}-\theta\right) \theta_{x} v v_{x}\right|}{\theta}\right] d x d s \\
& =I_{2}^{(1)}+I_{2}^{(2)}
\end{aligned}
$$

where

$$
\begin{aligned}
I_{2}^{(1)} & \leq C \int_{0}^{t} \int_{0}^{1}(1+\theta)^{r+1}\left|u_{x} v v_{x}\right| d x d s \\
& \leq C B(t)^{3 \beta / 2}, \\
I_{2}^{(2)} \leq & C \int_{0}^{t} \int_{0}^{1} \frac{(1+\theta)^{r}\left|\left(T_{0}-\theta\right) \theta_{x} v v_{x}\right|}{\theta} d x d s \\
& \leq \frac{1}{8} A(t)+C B(t)^{\max (r-q, 0)+\beta}
\end{aligned}
$$


or

$$
\begin{aligned}
I_{2}^{(2)} & \leq C \int_{0}^{t} \int_{0}^{1} \frac{(1+\theta)^{r+1}\left|\theta_{x} v v_{x}\right|}{\theta} d x d s \\
& \leq C\left(\int_{0}^{t} V(s) d s\right)^{1 / 2}\left(\int_{0}^{t} \int_{0}^{1}(1+\theta)^{2 r+2-q} v^{2} v_{x}^{2} d x d s\right)^{1 / 2} \\
& \leq C B(t)^{\left(\beta+\beta_{1}\right) / 2}
\end{aligned}
$$

or

$$
\begin{aligned}
I_{2}^{(2)} & \leq C\left(\int_{0}^{t} \int_{0}^{1}(1+\theta)^{2 r+2-q} v^{2} v_{x}^{2} d x d s\right)^{1 / 2} \\
& \leq C B(t)^{\delta / 2}\left(\int_{0}^{t} \int_{0}^{1}(1+\theta)^{2 m} v^{2} v_{x}^{2} d x d s\right)^{1 / 2} \\
& \leq C B(t)^{\left(2 \delta+3 \beta_{4}\right) / 4}
\end{aligned}
$$

which, combined with (2.39) and (2.40), implies

$$
I_{2}^{(2)} \leq \frac{1}{8} A(t)+C B(t)^{\beta_{10}} .
$$

By Lemmas 2.1-2.8, we get

$$
\begin{aligned}
I_{3} & \leq C \int_{0}^{t} \int_{0}^{1} \frac{\left(1+\theta^{q}\right)\left|\left(T_{0}-\theta\right) v v_{x} \theta_{x}\right|}{\theta} d x d s \\
& \leq \frac{1}{8} A(t)+C B(t)^{\max (q-r .0)+\beta}
\end{aligned}
$$

or

$$
\begin{aligned}
I_{3} & \leq C \int_{0}^{t} \int_{0}^{1} \frac{(1+\theta)^{q+1}\left|v v_{x} \theta_{x}\right|}{\theta} d x d s \\
& \leq C\left(\int_{0}^{t} V(s) d s\right)^{1 / 2}\left(\int_{0}^{t} \int_{0}^{1}(1+\theta)^{q+2} v^{2} v_{x}^{2} d x d s\right)^{1 / 2} \\
& \leq C B(t)^{(q+2+\beta) / 2}
\end{aligned}
$$

or

$$
\begin{aligned}
I_{3} & \leq C\left(\int_{0}^{t} \int_{0}^{1}(1+\theta)^{q+2} v^{2} v_{x}^{2} d x d s\right)^{1 / 2} \\
& \leq C B(t)^{\max (1-r .0) / 2}\left(\int_{0}^{t} \int_{0}^{1}(1+\theta)^{2 m} v^{2} v_{x}^{2} d x d s\right)^{1 / 2} \\
& \leq C B(t)^{\left(2 \max (1-r .0)+3 \beta_{4}\right) / 4}
\end{aligned}
$$

which, together with (2.42) and (2.43), gives

$$
I_{3} \leq \frac{1}{8} A(t)+C B(t)^{\beta_{11}} .
$$


Similarly,

$$
\begin{aligned}
I_{4} & \leq C\left|\int_{0}^{t} \int_{0}^{1} \frac{\left(T_{0}-\theta\right) k(u, \theta) \theta_{x}}{\theta}\left[-p(u, \theta) u_{x}+p_{\theta}\left(\theta-T_{0}\right) u_{x}\right] d x d s\right| \\
& \leq C \int_{0}^{t} \int_{0}^{1} \frac{(1+\theta)^{q+r+1}\left|\left(T_{0}-\theta\right) \theta_{x} u_{x}\right|+\left(T_{0}-\theta\right)^{2}(1+\theta)^{q+r}\left|u_{x} \theta_{x}\right|}{\theta} d x d s \\
& \leq \frac{1}{16} A(t)+C \int_{0}^{t} \int_{0}^{1}(1+\theta)^{2 m+1} u_{x}^{2} d x d s \\
& \quad+\int_{0}^{t} \int_{0}^{1} \frac{\left(T_{0}-\theta\right)^{2}(1+\theta)^{q+r}}{\theta}\left(\frac{\theta_{x}^{2}}{16 \theta}+C \theta u_{x}^{2}\right) d x d s \\
& \leq \frac{1}{8} A(t)+C B(t)^{\beta_{12}} .
\end{aligned}
$$

Therefore, (2.29) follows from (2.32)-(2.45).

Corollary 2.2 .

$$
\int_{0}^{t} \int_{0}^{1}(1+\theta)^{q+r} \theta_{x}^{2} d x d s \leq C B(t)^{\beta_{13}}, \quad \forall t>0
$$

with $\beta_{13}=\max \left(\beta_{8}, r\right)$.

Proof. Denote

$$
\tilde{\theta}=\theta-T_{0} \ln \theta
$$

Then

$$
\tilde{\theta}_{x}=\frac{\left(\theta-T_{0}\right) \theta_{x}}{\theta}, \quad \theta_{x}=\tilde{\theta}_{x}+T_{0} \frac{\theta_{x}}{\theta} .
$$

Thus it follows from Lemma 2.1, Lemma 2.10, (2.47) and Young's inequality that

$$
\begin{aligned}
\int_{0}^{t} \int_{0}^{1}(1+\theta)^{q+r} \theta_{x}^{2} d x d s & \leq C \int_{0}^{t} \int_{0}^{1}\left[(1+\theta)^{q+r} \tilde{\theta}_{x}^{2}+\frac{(1+\theta)^{q+r} \theta_{x}^{2}}{\theta^{2}}\right] d x d s \\
& \leq C B(t)^{\beta_{8}}+C B(t)^{r} \int_{0}^{t} V(s) d s \\
& \leq C B(t)^{\beta_{13}}
\end{aligned}
$$

which implies (2.46).

LEMma 2.9 .

$$
\begin{gathered}
\int_{0}^{t} \int_{0}^{1}(1+\theta)^{2 m+1} v_{x}^{2} d x d s \leq C B(t)^{\beta_{14}}, \quad \forall t>0 \\
\int_{0}^{t} \int_{0}^{1}(1+\theta)^{q+1}\left|v_{x}\right|^{3} d x d s \leq C B(t)^{\beta_{15}}, \quad \forall t>0 \\
\int_{0}^{t} \int_{0}^{1}(1+\theta)^{q-r} v_{x}^{4} d x d s \leq C B(t)^{\beta_{16}}, \quad \forall t>0
\end{gathered}
$$


where

$$
\begin{aligned}
\beta_{14} & =\max \left(\beta_{4}+1,2 m+1+\beta\right), \\
\beta_{15} & =\min \left(\beta_{17}, \beta_{6}+q+1\right), \\
\beta_{16} & =\min \left[q_{2}+\left(3 \beta_{4}+\beta_{5}\right) / 2, \max (q-r, 0)+\beta_{4}+\left(\beta+\beta_{5}\right) / 2\right], \\
\beta_{17} & =q_{1}+\left(5 \beta_{4}+\beta_{5}\right) / 4, \\
q_{1} & =\max [(q+1-3 r) / 4,0], \\
q_{2} & =\max [(q-3 r-1) / 2,0] .
\end{aligned}
$$

Proof. It is obvious from Lemma 2.3 and Lemma 2.8 that

$$
\int_{0}^{t} \int_{0}^{1}(1+\theta)^{2 m+1} v_{x}^{2} d x d s \leq C B(t)^{\beta_{4}+1}
$$

and

$$
\int_{0}^{t} \int_{0}^{1}(1+\theta)^{2 m+1} v_{x}^{2} d x d s \leq C B(t)^{2 m+1+\beta}
$$

which result in (2.48).

The interpolation inequality and Lemmas $2.1-2.8$ give

$$
\begin{aligned}
\int_{0}^{t} \int_{0}^{1}(1+\theta)^{q+1}\left|v_{x}\right|^{3} d x d s & \\
\leq & C B(t)^{q_{1}} \int_{0}^{t} \int_{0}^{1}(1+\theta)^{3 m / 2}\left|v_{x}\right|^{3} d x d s \\
\leq & C B(t)^{q_{1}}\left[\int_{0}^{t} \int_{0}^{1}\left|v_{x}\right|^{3} d x d s+\int_{0}^{t} \int_{0}^{1} V^{3 / 4}(s)\left|v_{x}\right|^{3} d x d s\right] \\
\leq & C B(t)^{q_{1}}\left[\sup _{0 \leq s \leq t}\left\|v_{x}\right\|\left(\int_{0}^{t}\left\|v_{x}\right\|^{2} d s\right)^{3 / 4}\left(\int_{0}^{t}\left\|v_{x x}\right\|^{2} d s\right)^{1 / 4}\right. \\
& +\sup _{0 \leq s \leq t}\left\|v_{x}\right\| \int_{0}^{t}\left\|v_{x}\right\|^{2} d s+\sup _{0 \leq s \leq t}\left\|v_{x}\right\|^{5 / 2}\left(\int_{0}^{t}\left\|v_{x x}\right\|^{2} d s\right)^{1 / 4} \\
\leq & \left.C B(t)^{\beta_{17}}+\sup _{0 \leq s \leq t}\left\|v_{x}\right\|^{5 / 2}\left(\int_{0}^{t}\left\|v_{x}\right\|^{2} d s\right)^{1 / 4}\right]
\end{aligned}
$$

and

$$
\begin{aligned}
\int_{0}^{t} \int_{0}^{1}(1+\theta)^{q+1}\left|v_{x}\right|^{3} d x d s & \leq C B(t)^{q+1} \int_{0}^{t}\left\|v_{x}\right\|_{L^{3}}^{3} d s \\
& \leq C B(t)^{q+1+\beta_{6}}
\end{aligned}
$$

which, combined with (2.51), yields (2.49). 
Similarly,

$$
\begin{aligned}
& \int_{0}^{t} \int_{0}^{1}(1+\theta)^{q-r} v_{x}^{4} d x d s \\
& \leq C B(t)^{q_{2}} \int_{0}^{t} \int_{0}^{1}(1+\theta)^{m} v_{x}^{4} d x d s \\
& \leq C B(t)^{q_{2}}\left[\int_{0}^{t}\left\|v_{x}\right\|_{L^{4}}^{4} d s+\int_{0}^{t} V^{1 / 2}(s)\left\|v_{x}\right\|_{L^{4}}^{4} d s\right] \\
& \leq C B(t)^{q_{2}}\left[\int_{0}^{t}\left(\left\|v_{x}\right\|^{3}\left\|v_{x x}\right\|+\left\|v_{x}\right\|^{4}\right) d s\right. \\
& \left.+\int_{0}^{t} V^{1 / 2}(s)\left\|v_{x}\right\|^{3}\left\|v_{x x}\right\| d s+\int_{0}^{t} V^{1 / 2}(s)\left\|v_{x}\right\|^{4} d s\right] \\
& \left.\leq C B(t)^{q_{2}+\left(3 \beta_{4}+\beta_{5}\right) / 2}+\sup _{0 \leq s \leq t}\left\|v_{x}\right\|^{3}\left(\int_{0}^{t} V(s) d s\right)^{1 / 2}\left(\int_{0}^{t}\left\|v_{x}\right\|^{2} d s\right)^{1 / 2}\right] \\
& \leq C B(t)^{q_{2}}\left[\sup _{0 \leq s \leq t}\left\|v_{x}\right\|^{2}\left(\int_{0}^{t}\left\|v_{x}\right\|^{2} d s\right)^{1 / 2}\left(\int_{0}^{t}\left\|v_{x x}\right\|^{2} d s\right)^{1 / 2}\left\|\sup _{0 \leq s \leq t}\right\| v_{x}\left\|^{2} \int_{0}^{t}\right\| v_{x}\left\|^{2} d s+\sup _{0 \leq s \leq t}\right\| v_{x} \|^{3}\left(\int_{0}^{t} V(s) d s\right)^{1 / 2}\left(\int_{x x} \|^{2} d s\right)^{1 / 2}\right. \\
& +\leq
\end{aligned}
$$

or

$$
\begin{aligned}
\int_{0}^{t} \int_{0}^{1}(1+\theta)^{q-r} v_{x}^{4} d x d s & \leq C B(t)^{\max (q-r, 0)} \int_{0}^{t}\left\|v_{x}\right\|_{L^{4}}^{4} d s \\
& \leq C B(t)^{\max (q-r, 0)+\left(2 \beta_{4}+\beta+\beta_{5}\right) / 2}
\end{aligned}
$$

with $\beta_{5} \geq \beta_{4} \geq 3 \beta$, which and (2.52) lead to (2.50).

LEMMA 2.10 .

$$
\int_{0}^{1}(1+\theta)^{2 q} \theta_{x}^{2} d x+\int_{0}^{t} \int_{0}^{1}(1+\theta)^{q+r} \theta_{t}^{2} d x d s \leq C B(t)^{\beta_{18}}, \quad \forall t>0
$$


where

$$
\begin{aligned}
\beta_{19}= & {\left[\max (3 q+2-r, 0)+\beta_{13}+\beta_{5}\right] / 2, } \\
\beta_{20}= & \min \left[\beta_{19},\left(3 q+4+\beta_{5}\right) / 2\right], \\
\beta_{21}= & \max \left[2 \max (q-r, 0)+2 \beta+\beta_{13}, \max (q-r, 0)+\beta+\left(\beta_{13}+\beta_{14}\right) / 2,\right. \\
& \left.\max (q-r, 0)+\beta+\left(\beta_{13}+\beta_{16}\right) / 2\right], \\
\beta_{22}= & \max [\max (q-r, 0)+q+2+\beta, 2 \max (q-r, 0)+r+2+2 \beta, \\
& \left.\max (q-r, 0)+\beta+\left(r+2+\beta_{14}\right) / 2, \max (q-r, 0)+\beta+\left(r+2+\beta_{16}\right) / 2\right], \\
\beta_{23}= & \min \left(\beta_{21}, \beta_{22}\right), \\
\beta_{24}= & \max \left[\left(\max (q-r, 0)+\beta_{7}+\beta_{13}\right) / 2,\left(2 \beta_{7}+\beta_{13}\right) / 3,\right. \\
& \left.\left(2 \beta_{7}+\beta_{13}+\beta_{14}\right) / 4,\left(2 \beta_{7}+\beta_{13}+\beta_{16}\right) / 4\right], \\
\beta_{25}= & \max \left[\left(q+2+\beta_{7}\right) / 2,\left(2 \beta_{7}+r+2\right) / 3,\left(2 \beta_{7}+r+2+\beta_{14}\right) / 4,\right. \\
& \left.\left(2 \beta_{7}+r+2+\beta_{16}\right) / 4\right], \\
\beta_{26}= & \min \left(\beta_{24}, \beta_{25}\right), \\
\beta_{18}= & \max \left[\beta_{14}, \beta_{15}, \beta_{16}, \beta_{20}, \beta_{23}, \beta_{26}\right] .
\end{aligned}
$$

Proof. Let

$$
\begin{gathered}
H(x, t)=H(u, \theta)=\int_{0}^{\theta} k(u, \xi) d \xi \\
X(t)=\int_{0}^{t} \int_{0}^{1}(1+\theta)^{q+r} \theta_{t}^{2} d x d s, \quad Y(t)=\int_{0}^{1}(1+\theta)^{2 q} \theta_{x}^{2} d x .
\end{gathered}
$$

Then it is easy to verify that

$$
\begin{aligned}
H_{t} & =H_{u} v_{x}+k \theta_{t}, \\
H_{x t} & =\left[k \theta_{x}\right]_{t}+H_{u} v_{x x}+H_{u u} v_{x} u_{x}+k_{u} u_{x} \theta_{t} .
\end{aligned}
$$

Multiplying (1.3) by $H_{t}$ and integrating the resultant over $Q_{t}$ results in

$$
\begin{gathered}
\int_{0}^{t} \int_{0}^{1}\left(e_{\theta} \theta_{t}+\theta p_{\theta} v_{x}-\mu v_{x}^{2}\right) H_{t} d x d s+\int_{0}^{t} \int_{0}^{1} k \theta_{x} H_{t x} d x d s \\
-\int_{0}^{t}\left(k \theta_{x} H_{t}\right)(1, s) d s+\int_{0}^{t}\left(k \theta_{x} H_{t}\right)(0, s) d s=0 .
\end{gathered}
$$

But we know from (1.25)-(1.29) that

$$
\left|H_{u}\right|+\left|H_{u u}\right| \leq C(1+\theta)^{q+1} .
$$

Now we estimate each term in (2.54) by using (1.25)-(1.29) and (2.55), Lemmas 2.1-2.10 and Corollaries 2.1-2.2.

By Lemmas 2.1-2.9 and Corollary 2.2, we can see that

$$
\int_{0}^{t} \int_{0}^{1} e_{\theta} \theta_{t} H_{t} d x d s \geq C_{0} X(t)-C B(t)^{\beta_{14}}
$$




$$
\begin{aligned}
\mid \int_{0}^{t} & \int_{0}^{1}\left(\theta p_{\theta} v_{x}-\mu v_{x}^{2}\right) H_{t} d x d s \mid \\
\leq & C \int_{0}^{t} \int_{0}^{1}\left[(1+\theta)^{q+r+2} v_{x}^{2}+(1+\theta)^{q+1}\left|v_{x}\right|^{3}\right. \\
& \left.+(1+\theta)^{q+r+1}\left|v_{x} \theta_{t}\right|+\left(1+\theta^{q}\right) v_{x}^{2}\left|\theta_{t}\right|\right] d x d s \\
& \leq \frac{C_{0}}{8} X(t)+C B(t)^{\beta_{14}}+C B(t)^{\beta_{15}}+C B(t)^{\beta_{16}},
\end{aligned}
$$

$$
\int_{0}^{t} \int_{0}^{1} k \theta_{x}\left(k \theta_{x}\right)_{t} d x d s \geq C Y(t)-C,
$$

$$
\begin{aligned}
\mid \int_{0}^{t} & \int_{0}^{1} k \theta_{x}\left(H_{u} v_{x x}+H_{u u} v_{x} u_{x}\right) d x d s \mid \\
\leq & C \int_{0}^{t} \int_{0}^{1}\left[(1+\theta)^{2 q+1}\left|\theta_{x}\right|\left(\left|v_{x x}\right|+\left|v_{x} u_{x}\right|\right)\right] d x d s \\
\leq & C B(t)^{\max (3 q+2-r, 0) / 2}\left(\int_{0}^{t} \int_{0}^{1}(1+\theta)^{q+r} \theta_{x}^{2} d x d s\right)^{1 / 2} \\
& \times\left[B(t)^{\beta_{5} / 2}+\sup _{0 \leq s \leq t}\left\|u_{x}\right\|\left(\int_{0}^{t}\left\|v_{x}\right\|_{L^{\infty}}^{2} d s\right)^{1 / 2}\right] \\
\leq & \left.C B(t)^{\beta_{19}}\right]
\end{aligned}
$$

with $\beta_{5} \geq \beta+\beta_{7}$ and $\beta_{5} \geq \beta_{4} \geq 3 \beta$.

Similarly,

$$
\begin{aligned}
& \left|\int_{0}^{t} \int_{0}^{1} k \theta_{x}\left(H_{u} v_{x x}+H_{u u} v_{x} u_{x}\right) d x d s\right| \\
& \quad \leq C\left(\int_{0}^{t} \int_{0}^{1}(1+\theta)^{4 q+2} \theta_{x}^{2} d x d s\right)^{1 / 2}\left[\left(\int_{0}^{t}\left\|v_{x x}\right\|^{2} d s\right)^{1 / 2}+\left(\int_{0}^{t}\left\|v_{x} u_{x}\right\|^{2} d s\right)^{1 / 2}\right] \\
& \quad \leq C B(t)^{(3 q+4) / 2}\left(\int_{0}^{t} V(s) d s\right)^{1 / 2}\left[B(t)^{\beta_{5} / 2}+B(t)^{\left(\beta+\beta_{7}\right) / 2}\right] \\
& \quad \leq C B(t)^{\left(3 q+4+\beta_{5}\right) / 2}
\end{aligned}
$$

which, together with (2.59), gives

$$
\left|\int_{0}^{t} \int_{0}^{1} k \theta_{x}\left(H_{u} v_{x x}+H_{u u} v_{x} u_{x}\right) d x d s\right| \leq C B(t)^{\beta_{20}}
$$


On the other hand, by Lemmas $2.1-2.10$, we can easily see that

$$
\begin{aligned}
\mid \int_{0}^{t} & \int_{0}^{1} k \theta_{x} k_{u} u_{x} \theta_{t} d x d s \mid \\
\leq & C \int_{0}^{t} \int_{0}^{1}(1+\theta)^{q}\left|k \theta_{x} u_{x} \theta_{t}\right| d x d s \\
\leq & \frac{C_{0}}{8} X(t)+C \int_{0}^{t} \int_{0}^{1}\left(k \theta_{x}\right)^{2}(1+\theta)^{q-r} u_{x}^{2} d x d s \\
\leq & \frac{C_{0}}{8} X(t)+C B(t)^{\max (q-r, 0)+\beta} \int_{0}^{t}\left\|k \theta_{x}\right\|_{L^{\infty}}^{2} d s \\
\leq & \frac{C_{0}}{8} X(t)+C B(t)^{\max (q-r, 0)+\beta} \int_{0}^{t}\left[\left\|k \theta_{x}\right\|^{2}+\int_{0}^{1}\left|k \theta_{x}\left(k \theta_{x}\right)_{x}\right| d x\right] d s \\
\leq & \frac{C_{0}}{8} X(t)+C B(t)^{\max (q-r, 0)+\beta}\left[B(t)^{\max (q-r, 0)} \int_{0}^{t} \int_{0}^{1}(1+\theta)^{q+r} \theta_{x}^{2} d x d s\right. \\
& \left.+\left(\int_{0}^{t} \int_{0}^{1}(1+\theta)^{q+r} \theta_{x}^{2} d x d s\right)^{1 / 2}\left(\int_{0}^{t} \int_{0}^{1}(1+\theta)^{q-r}\left|\left(k \theta_{x}\right)_{x}\right|^{2} d x d s\right)^{1 / 2}\right] \\
\leq & \frac{C_{0}}{8} X(t)+C B(t)^{2 \max (q-r, 0)+\beta+\beta_{13}}+C B(t)^{\max (q-r, 0)+\beta+\beta_{13} / 2} \\
& \times\left\{X(t)+\int_{0}^{t} \int_{0}^{1}\left[(1+\theta)^{q+r+2} v_{x}^{2}+(1+\theta)^{q-r} v_{x}^{4}\right] d x d s\right\}^{1 / 2} \\
\leq & \frac{C_{0}}{4} X(t)+C B(t)^{\beta_{21}} \cdot(2.61
\end{aligned}
$$

But we also know that

$$
\begin{aligned}
&\left|\int_{0}^{t} \int_{0}^{1} k \theta_{x} k_{u} u_{x} \theta_{t} d x d s\right| \leq \frac{C_{0}}{8} X(t)+C \int_{0}^{t} \int_{0}^{1}\left(k \theta_{x}\right)^{2}(1+\theta)^{q-r} u_{x}^{2} d x d s \\
& \leq \frac{C_{0}}{8} X(t)+C B(t)^{\max (q-r, 0)+\beta} \\
& \quad \times\left[\int_{0}^{t} \int_{0}^{1}(1+\theta)^{2 q} \theta_{x}^{2} d x d s+\int_{0}^{t} \int_{0}^{1}(1+\theta)^{q}\left|\theta_{x}\right|\left|\left(k \theta_{x}\right)_{x}\right| d x d s\right] \\
& \leq \frac{C_{0}}{8} X(t)+C B(t)^{\max (q-r, 0)+\beta}\left[B(t)^{q+2} \int_{0}^{t} V(s) d s\right. \\
&\left.\quad+\left(\int_{0}^{t} V(s) d s\right)^{1 / 2}\left(\int_{0}^{t} \int_{0}^{1} \theta^{2}(1+\theta)^{q}\left|\left(k \theta_{x}\right)_{x}\right|^{2} d x d s\right)^{1 / 2}\right] \\
& \leq \frac{C_{0}}{8} X(t)+C B(t)^{\max (q-r, 0)+q+2+\beta} \\
&+C B(t)^{\max (q-r, 0)+\beta+(r+2) / 2}\left(\int_{0}^{t} \int_{0}^{1}(1+\theta)^{q-r}\left|\left(k \theta_{x}\right)_{x}\right|^{2} d x d s\right)^{1 / 2} \\
& \leq \frac{C_{0}}{4} X(t)+C B(t)^{\beta_{22}} .
\end{aligned}
$$


Hence (2.61) and (2.62) imply

$$
\left|\int_{0}^{t} \int_{0}^{1} k \theta_{x} k_{u} u_{x} \theta_{t} d x d s\right| \leq \frac{C_{0}}{4} X(t)+C B(t)^{\beta_{23}}
$$

where $\beta_{23}=\min \left(\beta_{21}, \beta_{22}\right)$.

For $\tilde{\eta}=0$ or 1 , we have from (1.1), (1.6), (1.29), and Lemmas 2.1-2.10,

$$
\begin{aligned}
\left|H_{t}(\tilde{\eta}, t)\right|=\left|\left(H_{u} v_{x}\right)(\tilde{\eta}, t)\right|=\left|\int_{0}^{T_{0}}(k(u(\tilde{\eta}, t), \xi))_{u} d \xi v_{x}(\tilde{\eta}, t)\right| \\
\leq C\left|v_{x}(\tilde{\eta}, t)\right| \leq C\left\|v_{x}\right\|_{L^{\infty}} \\
\left|\int_{0}^{t}\left(k \theta_{x} H_{t}\right)(\tilde{\eta}, s) d s\right| \leq C\left(\int_{0}^{t}\left\|v_{x}\right\|_{L^{\infty}}^{2} d s\right)^{1 / 2}\left(\int_{0}^{t}\left\|k \theta_{x}\right\|_{L^{\infty}}^{2} d s\right)^{1 / 2} \\
\leq C B(t)^{\beta_{7} / 2}\left\{\int_{0}^{t}\left[\left\|k \theta_{x}\right\|^{2}+\int_{0}^{1}\left|k \theta_{x}\left(k \theta_{x}\right)_{x}\right| d x\right] d s\right\}^{1 / 2} \int_{0}^{t} \int_{0}^{1}(1+\theta)^{q+r} \theta_{x}^{2} d x d s \\
\leq C B(t)^{\beta_{7} / 2}\left\{B(t)^{\max (q-r, 0)} \int^{1 / 2}\left[\int_{0}^{t} \int_{0}^{1}(1+\theta)^{q-r}\left|\left(k \theta_{x}\right)_{x}\right|^{2} d x d s\right]^{1 / 2}\right\}^{1 / 2} \\
\quad+\left(\int_{0}^{t} \int_{0}^{1}(1+\theta)^{q+r} \theta_{x}^{2} d x d s\right)^{\left(2 \beta_{7}+\beta_{13}\right) / 4} \\
\leq C B(t)^{\left(\max (q-r, 0)+\beta_{7}+\beta_{13}\right) / 2}+C B(t)^{(2)} \\
\times\left[X(t)+B(t)^{\beta_{14}}+B(t)^{\beta_{16}}\right]^{1 / 4} \\
\leq \frac{C_{0}}{8} X(t)+C B(t)^{\beta_{24}} \cdot
\end{aligned}
$$

Similarly,

$$
\begin{aligned}
&\left|\int_{0}^{t}\left(k \theta_{x} H_{t}\right)(\tilde{\eta}, s) d s\right| \\
& \leq C B(t)^{\beta_{7} / 2}\left[B(t)^{q+2} \int_{0}^{t} V(s) d s\right. \\
&\left.+\left(\int_{0}^{t} V(s) d s\right)^{1 / 2}\left(\int_{0}^{t} \int_{0}^{1} \theta^{2}(1+\theta)^{q}\left|\left(k \theta_{x}\right)_{x}\right|^{2} d x d s\right)^{1 / 2}\right]^{1 / 2} \\
& \leq C B(t)^{\left(\beta_{7}+q+2\right) / 2}+C B(t)^{\left(2 \beta_{7}+r+2\right) / 4}\left[\int_{0}^{t} \int_{0}^{1}(1+\theta)^{q-r}\left|\left(k \theta_{x}\right)_{x}\right|^{2} d x d s\right]^{1 / 4} \\
& \leq C B(t)^{\left(\beta_{7}+q+2\right) / 2}+C B(t)^{\left(2 \beta_{7}+r+2\right) / 4}\left[X(t)+B(t)^{\beta_{14}}+B(t)^{\beta_{16}}\right]^{1 / 4} \\
& \leq \frac{C_{0}}{8} X(t)+C B(t)^{\beta_{25}} .
\end{aligned}
$$


Thus (2.64) and (2.65) give

$$
\mid \int_{0}^{t}\left(k \theta_{x} H_{t}(\tilde{\eta}, s) d s \mid \leq \frac{C_{0}}{8} X(t)+C B(t)^{\beta_{26}} .\right.
$$

Therefore, it follows from $(2.54),(2.56)-(2.58),(2.60),(2.63)$, and (2.66) that

$$
\begin{aligned}
X(t) & +Y(t) \\
& \leq C\left[B(t)^{\beta_{14}}+B(t)^{\beta_{15}}+B(t)^{\beta_{16}}+B(t)^{\beta_{20}}+B(t)^{\beta_{23}}+B(t)^{\beta_{26}}\right]
\end{aligned}
$$

which gives (2.53).

LEMMA 2.11 .

$$
\begin{gathered}
\|\theta\|_{L^{x}} \leq C, \quad \forall t>0 \\
\int_{0}^{1}\left(\theta_{x}^{2}+u_{x}^{2}+v_{x}^{2}\right) d x+\int_{0}^{t} \int_{0}^{1}\left[u_{x}^{2}+\theta_{x}^{2}+\theta_{t}^{2}+v_{t}^{2}+v_{x}^{2}+v_{x x}^{2}\right] d x d s \leq C, \quad \forall t>0 .
\end{gathered}
$$

Proof. By (1.6), Lemmas 2.1-2.10, we have

$$
\begin{aligned}
\|\theta\|_{L, x}^{q+(r+3) / 2} & \leq C+C \int_{0}^{1}\left|\theta^{q+(r+1) / 2} \theta_{x}\right| d x \\
& \leq C+C Y^{1 / 2}(t)\left(\int_{0}^{1} \theta^{r+1} d x\right)^{1 / 2} \\
& \leq C+C Y^{1 / 2}(t)
\end{aligned}
$$

which implies

$$
\|\theta\|_{L^{\infty}}^{2 q+r+3} \leq C+C Y(t) .
$$

Thus by (2.69), the Young inequality, Lemma 2.10, and noting that after a long complicated calculation, (1.22)-(1.24) imply $\beta_{18}<2 q+r+3$, we deduce

$$
\begin{aligned}
\|\theta\|_{L^{\infty}}^{2 q+r+3} & \leq C+C Y(t) \leq C B(t)^{\beta_{18}} \\
& \leq \frac{1}{2} \sup _{0 \leq s \leq t}\|\theta(s)\|_{L^{\infty}}^{2 q+r+3}+C
\end{aligned}
$$

which gives (2.67). The estimate (2.68) is the direct result of Lemmas 2.6-2.10.

REMARK 2.3. Similarly to the proof of (2.69), we can deduce that

$$
\|\theta\|_{L^{x}}^{2 q+2 r+4} \leq C B(t)^{\beta_{8}+\beta_{1 \gamma}} .
$$

Furthermore, we can know after a long complicated calculation that the range of $q$ obtained from (2.70) is smaller than that from (1.22)-(1.24).

3. Asymptotic behaviour. In this section, we show the results on asymptotic behaviour by use of an important lemma (Lemma 3.1) by Shen and Zheng in [23]. Though the proof of asymptotic behaviour is basically the same as that in [21], we still sketch it here for the convenience of the readers. 
Lemma 3.1 (Shen and Zheng [23]). Suppose that $y$ and $h$ are nonnegative functions on $[0,+\infty), y^{\prime}$ is locally integrable, and $y, h$ satisfy

$$
\begin{gathered}
\forall t>0: \quad y^{\prime}(t) \leq A_{1} y^{2}(t)+A_{2}+h(t), \\
\forall T>0: \quad \int_{0}^{T} y(s) d s \leq A_{3}, \quad \int_{0}^{T} h(s) d s \leq A_{4},
\end{gathered}
$$

with $A_{1}, A_{2}, A_{3}, A_{4}$ being positive constants independent of $t$ and $T$. Then for any $r>0$

$$
\forall t \geq 0: y(t+r) \leq\left(\frac{A_{3}}{r}+A_{2} r+A_{4}\right) e^{A_{1} A_{2}}
$$

Moreover,

$$
\lim _{t \rightarrow+\infty} y(t)=0
$$

LEMMA 3.2.

$$
\begin{aligned}
\int_{0}^{t}\left(\|p\|^{2}+\|\sigma\|^{2}\right) d s & \leq C, \quad \forall t>0, \\
\frac{d}{d t}\|p\|^{2} & \leq C\left(\|p\|^{2}+\left\|\theta_{t}\right\|^{2}+1\right), \quad \forall t>0, \\
\frac{d}{d t}\|v\|^{2} & \leq C\left(\left\|v_{t}\right\|^{2}+1\right), \quad \forall t>0 .
\end{aligned}
$$

Proof. Integrating (1.2) on $[0, x]$ and noting (1.6) give

$$
p(u, \theta)=\mu(u) v_{x}-\int_{0}^{x} v_{t} d y-\gamma v(0, t) .
$$

Hence from Lemmas 2.1-2.11, we have

$$
\int_{0}^{t}\|p\|^{2} d s \leq C \int_{0}^{t}\left(\left\|v_{x}\right\|^{2}+\left\|v_{t}\right\|^{2}+\gamma v^{2}(0, s)\right) d s \leq C
$$

which implies

$$
\int_{0}^{t}\|\sigma\|^{2} d s \leq C \int_{0}^{t}\left(\|p\|^{2}+\left\|v_{x}\right\|^{2}\right) d s \leq C .
$$

Clearly, we have

$$
\begin{aligned}
\frac{d}{d t}\|p\|^{2} & \leq C\left(\|p\|^{2}+\left\|p_{t}\right\|^{2}\right) \\
& \leq C\left(\|p\|^{2}+\left\|v_{x}\right\|^{2}+\left\|\theta_{t}\right\|^{2}\right) \\
& \leq C\left(1+\|p\|^{2}+\left\|\theta_{t}\right\|^{2}\right) .
\end{aligned}
$$

The proof of (3.3) is the same as that of (3.2). 
LEMMA 3.3 .

$$
\begin{aligned}
\frac{d}{d t}\left\|u_{x}\right\|^{2} & \leq\left\|v_{x x}\right\|^{2}+\left\|u_{x}\right\|^{2}, \quad \forall t>0, \\
\frac{d}{d t}\left\|\theta_{x}\right\|^{2}+C_{9} \int_{0}^{1}(1+\theta)^{q-r} \theta_{x x}^{2} d x & \leq C\left(\left\|v_{x x}\right\|^{2}+1\right), \quad \forall t>0, \\
\left\|\theta_{x}\right\|^{2}+\int_{0}^{t} \int_{0}^{1}(1+\theta)^{q-r} \theta_{x x}^{2} d x d s & \leq C, \quad \forall t>0 .
\end{aligned}
$$

Proof. Differentiating (1.1) with respect to $x$ and multiplying the resultant by $u_{x}$ yield the estimate (3.4).

Multiplying (1.3) by $e_{\theta}^{-1} \theta_{x x}$ and integrating the resultant on $[0,1]$ lead to

$$
\begin{gathered}
\frac{d}{d t}\left\|\theta_{x}\right\|^{2}+2 \int_{0}^{1} k \theta_{x x}^{2} e_{\theta} d x \\
\leq \varepsilon\left\|\theta_{x x}\right\|^{2}+C\left(\left\|v_{x}\right\|^{2}+\left\|v_{x}\right\|_{L^{4}}^{4}+\left\|\theta_{x}\right\|_{L^{4}}^{4}+\left\|u_{x} \theta_{x}\right\|^{2}\right) \\
\leq \varepsilon\left\|\theta_{x x}\right\|^{2}+C\left(\left\|v_{x}\right\|^{2}+\left\|v_{x}\right\|^{3}\left\|v_{x x}\right\|+\left\|v_{x}\right\|^{4}\right. \\
\left.\quad+\left\|\theta_{x}\right\|^{3}\left\|\theta_{x x}\right\|+\left\|\theta_{x}\right\|^{4}+\left\|\theta_{x}\right\|_{L^{\infty}}^{2}\right) \\
\leq 2 \varepsilon\left\|\theta_{x x}\right\|^{2}+C\left(\left\|v_{x}\right\|^{2}+\left\|v_{x x}\right\|^{2}+\left\|\theta_{x}\right\|^{2}\right) .
\end{gathered}
$$

Hence for small $\varepsilon$, we have

$$
\begin{aligned}
\frac{d}{d t}\left\|\theta_{x}\right\|^{2}+C_{9} \int_{0}^{1}(1+\theta)^{q-r} \theta_{x x}^{2} d x & \leq C\left(\left\|v_{x}\right\|^{2}+\left\|v_{x x}\right\|^{2}+\left\|\theta_{x}\right\|^{2}\right) \\
& \leq C\left(\left\|v_{x x}\right\|^{2}+1\right)
\end{aligned}
$$

which implies

$$
\left\|\theta_{x}\right\|^{2}+\int_{0}^{t} \int_{0}^{1}(1+\theta)^{q-r} \theta_{x x}^{2} d x d s \leq C+C \int_{0}^{t}\left(\left\|v_{x}\right\|^{2}+\left\|v_{x x}\right\|^{2}+\left\|\theta_{x}\right\|^{2}\right) d s \leq C .
$$

It is the most difficult to prove $\left\|v_{x}\right\| \rightarrow 0$ as $t \rightarrow \infty$; thus, the following lemma plays a very important role in proving this.

LEMMA 3.4.

$$
\begin{aligned}
\int_{0}^{t} Z(s) d s & \leq C, \quad \forall t>0, \\
\frac{d}{d t} Z(t) & \leq C\left(\left\|v_{x x}\right\|^{2}+\left\|\theta_{t}\right\|^{2}+\left\|v_{t}\right\|^{2}+1\right)
\end{aligned}
$$

where

$$
\begin{aligned}
Z(t):= & \frac{1}{2} \int_{0}^{1} \mu(v) v_{x}^{2} d x+\frac{\gamma}{2}\left(v^{2}(0, t)+v^{2}(1, t)\right)+p\left(u(0, t), T_{0}\right) v(0, t) \\
& \left.-p\left(u(1, t), T_{0}\right) v(1, t)\right)+C_{10}\left(\|p\|^{2}+\left\|\theta_{x}\right\|^{2}+\left\|u_{x}\right\|^{2}+\|v\|^{2}\right)
\end{aligned}
$$

with a sufficiently large positive constant $C_{10}$ such that

$$
Z(t) \geq C\left(\left\|v_{x}\right\|^{2}+\|v\|^{2}+\left\|\theta_{x}\right\|^{2}+\left\|u_{x}\right\|^{2}\right)+\frac{\gamma}{2}\left(v^{2}(0, t)+v^{2}(1, t)\right) .
$$


Proof. Denote

$$
w(t)=\frac{1}{2} \int_{0}^{1} \mu(u) v_{x}^{2} d x+\frac{\gamma}{2}\left(v^{2}(0, t)+v^{2}(1, t)\right)+p\left(u(0, t), T_{0}\right) v(0, t)-p\left(u(1, t), T_{0}\right) v(1, t) .
$$

Thus, multiplying $(1.2)$ by $v_{t}$, integrating the resultant over $[0,1]$, integrating by parts and noting $(2.19)-(2.20)$, give

$$
\begin{aligned}
\left\|v_{t}\right\|^{2}+ & w^{\prime}(t) \\
= & \frac{1}{2} \int_{0}^{1} \mu^{\prime}(u) v_{x}^{3} d x-\int_{0}^{1} p_{x} v_{t} d x-p_{t}\left(u(0, t), T_{0}\right) v(0, t)+p_{t}\left(u(1, t), T_{0}\right) v(1, t) \\
\leq & C\left(\left\|v_{x}\right\|_{L^{3}}^{3}+\left\|u_{x}\right\|^{2}+\left\|\theta_{x}\right\|^{2}\right)+\frac{1}{2}\left\|v_{t}\right\|^{2} \\
& +\left|p_{u}\left(u(0, t), T_{0}\right) v_{x}(0, t) v(0, t)\right|+\left|p_{u}\left(u(1, t), T_{0}\right) v_{x}(1, t) v(1, t)\right| \\
\leq & C\left(\left\|v_{x}\right\|_{L^{3}}^{3}+\left\|u_{x}\right\|^{2}+\left\|\theta_{x}\right\|^{2}+\left\|v_{x}\right\|_{L^{\infty}}^{2}+\|v\|_{L^{\infty}}^{2}\right)+\frac{1}{2}\left\|v_{t}\right\|^{2},
\end{aligned}
$$

i.e.,

$$
w^{\prime}(t)+\frac{1}{2}\left\|v_{t}\right\|^{2} \leq C\left(\left\|v_{x}\right\|_{L^{3}}^{3}+\left\|u_{x}\right\|^{2}+\left\|\theta_{x}\right\|^{2}+\left\|v_{x}\right\|_{L^{\infty}}^{2}+\|v\|_{L^{\infty}}^{2}\right) .
$$

But due to (1.15) and using the mean-value theorem, we have

$$
p(u, \theta)=p_{u}\left(u, \theta_{1}\right)\left(u-\eta_{0}\right)+p_{\theta}\left(u_{1}, \theta_{1}\right)\left(\theta-T_{0}\right)
$$

where $\min \left(u, \eta_{0}\right) \leq u_{1} \leq \max \left(u, \eta_{0}\right)$ and $\min \left(\theta, T_{0}\right) \leq \theta_{1} \leq \max \left(\theta, T_{0}\right)$.

Hence from (1.26), we get

$$
\begin{gathered}
\left|u-\eta_{0}\right| \leq C\left(|p|+\left|\theta-T_{0}\right|\right), \\
\left\|u-\eta_{0}\right\| \leq C\left(\|p\|+\left\|\theta-T_{0}\right\|\right) \leq C\left(\|p\|+\left\|\theta_{x}\right\|\right) .
\end{gathered}
$$

By the Nirenberg inequality and (3.14)-(3.15), we have

$$
\begin{aligned}
\|p\|_{L^{\infty}} & \leq C\left(\left\|u-\eta_{0}\right\|_{L^{\infty}}+\left\|\theta-T_{0}\right\|_{L^{\infty}}\right) \\
& \leq C\left(\left\|u-\eta_{0}\right\|^{1 / 2}\left\|u_{x}\right\|^{1 / 2}+\left\|u-\eta_{0}\right\|+\left\|\theta_{x}\right\|\right) \\
& \leq C\left(\|p\|+\left\|\theta_{x}\right\|+\left\|u_{x}\right\|\right)
\end{aligned}
$$

and

$$
\|v\|_{L^{\infty}} \leq C\left(\|v\|^{1 / 2}\left\|v_{x}\right\|^{1 / 2}+\|v\|\right) \leq C\left(\|v\|+\left\|v_{x}\right\|\right)
$$

Thus,

$$
\|p\|_{L^{\infty}}\|v\|_{L^{\infty}} \leq \varepsilon\left\|v_{x}\right\|^{2}+C\left(\|v\|^{2}+\|p\|^{2}+\left\|u_{x}\right\|^{2}+\left\|\theta_{x}\right\|^{2}\right) .
$$

Clearly, we have

$$
\begin{gathered}
w(t) \geq C_{11}\left\|v_{x}\right\|^{2}+\frac{\gamma}{2}\left(v^{2}(0, t)+v^{2}(1, t)\right)-2\|p\|_{L^{\infty}}\|v\|_{L^{\infty}} \\
\geq\left(C_{11}-2 \varepsilon\right)\left\|v_{x}\right\|^{2}+\frac{\gamma}{2}\left(v^{2}(0, t)+v^{2}(1, t)\right) \\
-C_{12}\left(\|p\|^{2}+\|v\|^{2}+\left\|u_{x}\right\|^{2}+\left\|\theta_{x}\right\|^{2}\right) .
\end{gathered}
$$

Taking $\varepsilon>0$ sufficiently small in (3.19) $\left(2 \varepsilon<C_{11}\right)$ and taking $C_{10}>0$ sufficiently large in (3.9) $\left(C_{10}>C_{12}\right)$, give $(3.10)$. 
On the other hand, it is easy to get from (3.9) and (3.16)-(3.17)

$$
\begin{gathered}
Z(t) \leq C\left(\left\|v_{x}\right\|^{2}+\|p\|^{2}+\left\|\theta_{x}\right\|^{2}+\left\|u_{x}\right\|^{2}+\|p\|_{L^{x}}^{2}+\|v\|_{L^{x}}^{2}\right) \\
\quad+\frac{\gamma}{2}\left(v^{2}(0, t)+v^{2}(1, t)\right) \\
\leq C\left(\left\|v_{x}\right\|^{2}+\|p\|^{2}+\left\|\theta_{x}\right\|^{2}+\left\|u_{x}\right\|^{2}+\|v\|_{L^{x}}^{2}\right) \\
\quad+\frac{\gamma}{2}\left(v^{2}(0, t)+v^{2}(1, t)\right)
\end{gathered}
$$

which implies (3.7) by Lemmas 2.1-2.11.

From (3.9), (3.12) and Lemmas 3.1-3.2, we easily deduce

$$
\begin{aligned}
\frac{d}{d t} Z(t)= & w^{\prime}(t)+C_{10} \frac{d}{d t}\left(\|p\|^{2}+\left\|\theta_{x}\right\|^{2}+\left\|u_{x}\right\|^{2}+\|v\|^{2}\right) \\
\leq & C\left(\left\|v_{x}\right\|_{L^{3}}^{3}+\left\|u_{x}\right\|^{2}+\left\|\theta_{x}\right\|^{2}+\left\|v_{x}\right\|_{L^{x}}^{2}+\|v\|_{L^{x}}^{2}\right. \\
& \left.\quad+\|p\|^{2}+\left\|\theta_{t}\right\|^{2}+\left\|v_{x x}\right\|^{2}+\left\|v_{t}\right\|^{2}+1\right) \\
\leq & C\left(\left\|v_{x x}\right\|^{2}+\left\|\theta_{t}\right\|^{2}+\left\|v_{t}\right\|^{2}+1\right) .
\end{aligned}
$$

Lemma 3.5. As $t \rightarrow+\infty$, we have

$$
\begin{aligned}
\left\|u-\eta_{0}\right\|_{H^{1}} \rightarrow 0, \quad\left\|u_{x}\right\| \rightarrow 0, \quad\left\|u-\eta_{0}\right\|_{L^{x}} & \rightarrow 0, \\
\|v\|_{H^{1}} \rightarrow 0, \quad\left\|v_{x}\right\| & \rightarrow 0, \\
\left\|\theta-T_{0}\right\|_{H^{1}} \rightarrow 0, \quad\left\|\theta_{x}\right\| \rightarrow 0, \quad\left\|\theta-T_{0}\right\|_{L^{x}} & \rightarrow 0, \\
\|p\| \rightarrow 0, \quad \gamma\left(v^{2}(0, t)+v^{2}(1, t)\right) & \rightarrow 0, \\
\|p(u, \theta)\|_{H^{1}} \rightarrow 0, \quad\|\sigma(u, \theta)\| & \rightarrow 0,
\end{aligned}
$$

where $\left(\eta_{0}, 0, T_{0}\right)$ is the unique solution to the corresponding stationary problem to the problem (1.1)-(1.3), (1.5)-(1.6). Moreover, there exist positive constants $C_{1}, C_{2}, C_{3}$ such that for all $t \geq C_{1}$, it follows that

$$
\|v(t)\|_{H^{1}}+\left\|\theta(t)-T_{0}\right\|_{H^{1}}+\left\|u(t)-\eta_{0}\right\|_{H^{1}} \leq C_{2} \exp \left(-C_{3} t\right) .
$$

Proof. (3.21) is the direct consequence by applying Lemma 2.1 and Lemma 3.3. It is obvious that (3.22)-(3.26) are the consequence of (3.21). It is easy to verify that $\left(\eta_{0}, 0, T_{0}\right)$ is the unique solution to the corresponding stationary problem to the problem (1.1)-(1.3), (1.5)-(1.6).

Now since $\left(u(t)-\eta_{0}, v(t), \theta(t)-T_{0}\right)$ can be small in the $H^{1}$ norm for sufficiently large $t$, we can deduce the desired estimate (3.27) by the same method as that in [16].

Proofs of Theorem 1.1 and Theorem 2.1. By the standard argument (see [10]) and Lemmas 2.1-2.11, we complete the proof of Theorem 1.1 and Theorem 2.1.

REMARK 3.1. It follows from the proof of Lemmas 2.1-2.11 and Lemmas 3.1-3.4, Theorem 1.1 that all the constants in Lemmas 2.1-2.11 and Lemmas 3.1-3.4 depend only on the $H^{1}$ norm of the initial data $\left(u_{0}, v_{0}, \theta_{0}\right)$. Therefore, the following results of global existence, uniqueness and the same results of the asymptotic behaviour as Theorem 1.1 hold: If $\left(u_{0}, v_{0}, \theta_{0}\right) \in H^{1} \times H^{1} \times H^{1}$, the problem (1.1)-(1.3), (1.5)-(1.6) admits a 
unique generalized solution $(u(t), v(t), \theta(t))$ in the sense that $u \in L^{\infty}\left(0,+\infty ; H^{1}\right), u^{\prime} \in$ $L^{\infty}\left(0,+\infty ; L^{2}\right),(v, \theta) \in L^{\infty}\left(0,+\infty ; H^{1}\right) \cap L^{2}\left(0,+\infty ; H^{2}\right) \cap H^{1}\left(0,+\infty ; L^{2}\right)$. Moreover, (3.21)-(3.27) hold.

Acknowledgment. The author would like to thank his Ph.D. advisor Professor Songmu Zheng for his constant instruction and sincere help. The author is also grateful to Professor Song Jiang for his constant sincere help.

\section{REFERENCES}

[1] C. M. Dafermos, Global smooth solutions to the initial boundary value problem for the equations of one-dimensional nonlinear thermoviscoelasticity, SIAM J. Math. Anal. 13, 397-408 (1982)

[2] C. M. Dafermos and L. Hsiao, Global smooth thermomechanical processes in one-dimensional nonlinear thermoviscoelasticity, Nonlinear Anal. 6, 435-454 (1982)

[3] S. Jiang, Global large solutions to initial boundary value problems in one-dimensional nonlinear thermoviscoelasticity, Quart. Appl. Math. 51, 731-744 (1992)

[4] S. Jiang, On the initial boundary value problems for a viscous, heat-conducting, one-dimensional real gas, J. Differential Equations 110, 157-181 (1994)

[5] S. Jiang, Global spherically symmetric solutions to the equations of a viscous polytropic ideal gas in exterior domain, Comm. Math. Phys. 178, 339-374 (1996)

[6] S. Jiang, Large-time behaviour of solutions to the equations of a viscous polytropic ideal gas, Ann. Mat. Pura Appl. 175, 253-275 (1998)

[7] S. Jiang, Large-time behaviour of solutions to the equations of a one-dimensional viscous polytropic ideal gas in unbounded domains, Comm. Math. Phys. 200, 181-193 (1999)

[8] L. Hsiao and H. Jian, Asymptotic behaviour of solutions to the system of one-dimensional nonlinear thermoviscoelasticity, Chinese Ann. Math. Ser. B 19, 143-152 (1998)

[9] L. Hsiao and T. Luo, Large-time behaviour of solutions to the equations of one-dimensional nonlinear thermoviscoelasticity, Quart. Appl. Math. 56, 201-219 (1998)

[10] S. Kawashima and T. Nishida, Global solutions to the initial boundary value problems for the equations of one-dimensional motion of viscous polytropic gases, J. Math. Kyoto Univ. 21, 825-837 (1981)

[11] B. Kawohl, Global existence of large solutions to initial boundary value problems for a viscous, heat-conducting, one-dimensional real gas, J. Differential Equations 58, 76-103 (1985)

[12] A. V. Kazhikhov and V. V. Shelukhin, Unique global solution with respect to time of initial boundary value problems for one-dimensional equations of a viscous gas, J. Appl. Math. Meth. 41, 273-282 (1977)

[13] J. U. Kim, Global existence of solutions of the equations of one-dimensional thermoviscoelasticity with initial data in $B V$ and $L^{1}$, Ann. Scuola Norm. Sup. Pisa 10, 357-427 (1983)

[14] T. Nagasawa, On the one-dimensional motion of the polytropic ideal gas non-fixed on the boundary, J. Differential Equations 65, 49-67 (1986)

[15] T. Nagasawa, On the asymptotic behaviour of the one-dimensional motion of the polytropic ideal gas with stress-free condition, Quart. Appl. Math. 46, 665-679 (1988)

[16] M. Okada and S. Kawashima, On the equation of one-dimensional motion of compressible viscous fluids, J. Math. Kyoto Univ. 23, 55-71 (1983)

[17] Y. Qin, Global existence and asymptotic behaviour of solutions to a system of equations for a nonlinear one-dimensional viscous, heat-conducting real gas, Chinese Ann. Math. Ser. A 20 (3), 343-354 (1999)

[18] Y. Qin, Global existence and asymptotic behaviour of a viscous, heat-conductive, one-dimensional real gas with fixed and thermally insulated endpoints, Nonlinear Anal. (to appear)

[19] Y. Qin, Global existence and asymptotic behaviour for the solution to nonlinear viscous, heatconductive, one-dimensional real gas, Adv. Math. Sci. Appl. 10, 119-148 (2000)

[20] Y. Qin, Global existence and asymptotic behaviour for a viscous, heat-conductive, one-dimensional real gas with fixed and constant temperature boundary conditions, submitted to Differential and Integral Equations 
[21] Y. Qin, Asymptotic behaviour for global smooth solution to a one-dimensional nonlinear thermoviscoelastic system, J. Partial Differential Equations 12, 111-134 (1999)

[22] R. Racke and S. Zheng, Global existence and asymptotic behaviour in nonlinear thermoviscoelasticity, J. Differential Equations 1, 46-67 (1997)

[23] W. Shen and S. Zheng, On the coupled Cahn-Hilliard equations, Comm. Partial Differential Equations 18, 701-727 (1993)

[24] W. Shen and S. Zheng, Global smooth solutions to the Cauchy problem of equations of onedimensional nonlinear thermoviscoelasticity, Partial Differential Equations 2, 26-38 (1989)

[25] W. Shen, S. Zheng, and P. Zhu, Global existence and asymptotic behaviour of weak solutions to nonlinear thermoviscoelastic system with clamped boundary conditions, Quart. Appl. Math. 57, 93-116 (1999)

[26] J. Sprekels, S. Zheng, and P. Zhu, Asymptotic behaviour of the solutions to a Landau-Ginzburg system with viscosity for martensitic phase transitions in shape memory alloys, SIAM J. Math. Anal. 1, 69-84 (1998)

[27] S. Zheng, Nonlinear parabolic equations and hyperbolic-parabolic coupled systems, Pitman Monographs and Surveys in Pure and Applied Mathematics, Vol. 76, Longman Group Limited, London, 1995 